\title{
A hyperelastic model for soils with stress-induced and inherent anisotropy
}

\author{
Marcin Cudny ${ }^{1}$ (D) Katarzyna Staszewska ${ }^{1}$
}

Received: 3 August 2020 / Accepted: 4 February 2021 / Published online: 5 March 2021

(C) The Author(s) 2021

\begin{abstract}
In this paper, modelling of the superposition of stress-induced and inherent anisotropy of soil small strain stiffness is presented in the framework of hyperelasticity. A simple hyperelastic model, capable of reproducing variable stress-induced anisotropy of stiffness, is extended by replacement of the stress invariant with mixed stress-microstructure invariant to introduce constant inherent cross-anisotropic component. A convenient feature of the new model is low number of material constants directly related to the parameters commonly used in the literature. The proposed description can be incorporated as a small strain elastic core in the development of some more sophisticated hyperelastic-plastic models of overconsolidated soils. It can also be used as an independent model in analyses involving small strain problems, such as dynamic simulations of the elastic wave propagation. Various options and features of the proposed anisotropic hyperelastic model are investigated. The directional model response is compared with experimental data available in the literature.
\end{abstract}

Keywords Cross-anisotropy · Hyperelasticity · Overconsolidated soils - Small strain stiffness · Soil elasticity · Stiffness anisotropy

\section{Introduction}

An elastic stress-strain relation is the core of all the elastoplastic constitutive models. It describes the initial stiffness and influences substantially the modelled pre-failure behaviour of soils. In the case of natural soils, there are two important features of the initial elastic stiffness, namely barotropy and anisotropy. Barotropy is typically taken into account as the dependency of stiffness on the actual stress level that is represented by the mean stress $p$ or by one of the principal stress components $\sigma_{i}$. Models used in the geotechnical practice, e.g. [49], mostly incorporate the exponential empirical relation in the form proposed by Ohde [45] or Janbu [31]. In this relation, stress dependency of stiffness concerns elastic moduli in the isotropic

Marcin Cudny

mcud@pg.edu.pl

Katarzyna Staszewska

katstasz@pg.edu.pl

1 Faculty of Civil and Environmental Engineering, Gdańsk University of Technology, ul. Narutowicza 11/12, 80-233 Gdańsk, Poland
Hooke's law and the Poisson's ratio $v$ is assumed constant. This results in an isotropic hypoelastic relation which, of course, is dependent neither on the stress obliquity (e.g. $\eta=q / p$ or $\left.K=\sigma_{3} / \sigma_{1}\right)$ nor on the direction of loading.

Influence of the stress obliquity is often described as stress-induced anisotropy. In the case of stress-induced anisotropy, a directional distribution of stiffness changes depending on the current stress position in the principal stress space. Stress-induced anisotropy vanishes under isotropic stress conditions. However, stiffness of natural soils remains anisotropic even under isotropic stress conditions. This is due to the anisotropic microstructure of soil fabric that was developed as a result of deposition and diagenesis [23, 30, 39]. This component of anisotropy is described as inherent anisotropy. Its properties can be investigated only under isotropic stress conditions. In natural soils, the actual directional dependency of elastic stiffness is a superposition of both stress-induced and inherent anisotropy. Such situation is illustrated schematically in Fig. 1. It is an exemplary problem of the slope stability, in which the principal axes of stress $\mathbf{n}_{\boldsymbol{\sigma} i}$ and microstructure $x_{\mathrm{mi}}$ are generally not collinear. They are also different from the applied geometrical coordinate 


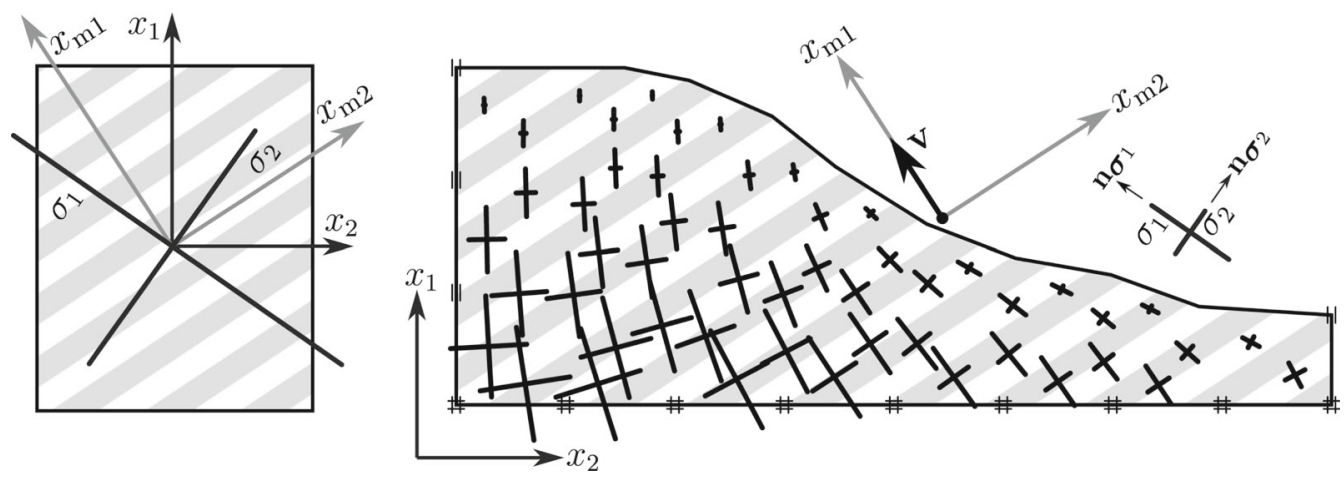

Fig. 1 Geometrical $x_{i}$, stress $\mathbf{n}_{\sigma i}$ and microstructural $x_{m i}$ principal axes in a representative soil element and in an exemplary boundary value problem of the slope stability analysis

system $x_{i}$ which usually corresponds to the direction of gravity.

A superposed anisotropy of the actual elastic stiffness is usually complex. Principal stress directions may rotate relative to the soil microstructure as a result of geotechnical processes (e.g. excavation, tunnel drilling). Hence, the directional dependency of stiffness changes with the angle between the principal axes of stress and of microstructure. In the standard laboratory tests, such as triaxial or oedometric compression, these systems are collinear and rotation of the principal stress directions is not possible. Contrarily to stress-induced anisotropy, inherent anisotropy of the elastic stiffness remains rather constant even after significant stress changes [32]. This seems to be evident in natural heavily overconsolidated clays. In reconstituted or moderately overconsolidated clays, inherent anisotropy may evolve due to irreversible straining as it is shown in experimental investigations by Mitaritonna et al. [40].

In the recent years, the importance of stiffness nonlinearity and anisotropy in the small and intermediate strain ranges has been shown in many analyses of practical cases in geotechnics [1, 3, 11, 16, 35, 42, 47]. Anisotropy of the elastic stiffness is also known to influence the yielding characteristics of soils in the undrained conditions [44, 50, 56].

A common type of inherent anisotropy is the crossanisotropy, also called the transverse isotropy. In this case, the elastic material properties are symmetric about axis that is normal to the plane of isotropy. In soils, the plane of isotropy is identified with the prevailed particle orientation or bedding planes. The symmetry axis, here indicated by the unit vector $\mathbf{v}$, is collinear with the deposition direction. This direction is usually vertical; however, it may be inclined due to geological processes, especially in the older overconsolidated clayey deposits, as depicted in Fig. 1.

In the classical linear elastic description, a cross-anisotropic stiffness requires the definition of five independent material constants [46]. Different sets of five independent cross-anisotropic parameters may be selected. Using notation with indices $h$ and $v$ to denote the horizontal and the vertical directions, respectively, these parameters are usually chosen as: $E_{v}$, Young's modulus in vertical direction; $E_{h}$, Young's modulus in horizontal direction; $v_{v h}$, Poisson's ratio for horizontal strain due to vertical strain; $v_{h h}$, Poisson's ratio for horizontal strain due to complementary horizontal strain; $G_{v h}$, shear modulus in vertical plane. This naming convention assumes the horizontal plane of isotropy. However, if vector $\mathbf{v}$ deviates from vertical direction, plane $h-h$ is also rotated relative to the horizon. Hence, one needs to note that indices $h$ and $v$ are associated with the cross-anisotropic microstructure.

Based on the experimental observations, Graham and Houlsby [21] proposed a simplification of the five-parameter cross-anisotropic stiffness. They reduced the number of material constants to three independent parameters leaving $E_{v}$ and $v_{h h}$, denoted by $E^{*}$ and $v^{*}$, respectively, and introducing the anisotropy coefficient $\alpha$ that imposes the following condition:

$\alpha=\sqrt{\frac{E_{h}}{E_{v}}}=\frac{v_{h h}}{v_{v h}}=\frac{G_{h h}}{G_{v h}}$,

where $G_{h h}$ is the shear modulus in the plane of isotropy:

$G_{h h}=\frac{E_{h}}{2\left(1+v_{h h}\right)}$.

Values of the anisotropy coefficient $\alpha$ in the overconsolidated soil deposits are generally higher than 1.0, which indicates higher stiffness in the plane of isotropy, i.e. $G_{h h}>G_{v h}$ and $E_{h}>E_{v}$. In normally consolidated or lightly overconsolidated soils, stiffness may be higher in the direction of symmetry axis [37, 55]; however, the opposite situation is also reported [9, 36]. Intensity of inherent cross-anisotropy, expressed as a deviation of $\alpha$ from 1.0 , is also observed to be higher in clayey deposits $[8,26]$ than in sands. This is due to the fact that the total 
anisotropy in sands is mostly dominated by stress-induced component [27, 34].

Mašín and Rott [38] refined the definition of $\alpha$ into three coefficients corresponding to the Young's and shear moduli, and the Poisson's ratios, respectively:

$$
\begin{aligned}
& \alpha_{E}=\frac{E_{h}}{E_{v}}, \\
& \alpha_{G}=\frac{G_{h h}}{G_{v h}}, \\
& \alpha_{v}=\frac{v_{h h}}{v_{v h}} .
\end{aligned}
$$

Additionally, to compare the anisotropy coefficients, they proposed two anisotropy exponents $x_{G E}$ and $x_{G v}$ :

$$
\begin{aligned}
& \alpha_{G}=\alpha_{E}^{x_{G E}}, \\
& \alpha_{G}=\alpha_{v}^{x_{G v}} .
\end{aligned}
$$

It follows that in the cross-anisotropic stiffness proposed in [21], the imposed values of anisotropy exponents are: $x_{G E}=0.5$ and $x_{G v}=1.0$. However, after careful inspection of the recent experimental data for natural clays, this is shown in [38] not to be necessarily valid and approximate average value of $x_{G E}=0.8$ is suggested. Due to difficulties in the measurement of the anisotropic Poisson's ratios, the values of $\alpha_{v}$ and $x_{G v}$ are characterised by large scatter; hence, further experimental investigations are needed. It is suggested in [38] that, for practical purposes, $x_{G v}=1.0$ should be adopted.

The classical linear cross-anisotropic stiffness is not stress-dependent. One should be careful when introducing an empirical barotropy of cross-anisotropic elastic parameters into this model. This is because of the constraints imposed on these parameters by the elastic energy conservation requirement [46].

The objective of this work is to present an elastic model capable of reproducing the stiffness barotropy, stress-induced anisotropy and inherent cross-anisotropy. For this purpose, we use the framework of hyperelasticity $[25,28,43]$. The proposed model is based on an improvement of the elastic potential function by Vermeer [51]. The new refined potential function is not dependent on the stress invariant allowing stress-induced anisotropy only but instead on the new mixed stress-microstructure invariant. The mixed invariant introduces inherent anisotropy component in a way proposed by Boehler and coworkers [5-7]. This method allows to control the values of anisotropy coefficients and the exponents defined in [38]. The initial works on the proposed refinement of Vermeer's elastic potential are reported in monograph [10] as a part of a hyperelastic-plastic model of overconsolidated fine-grained soils.
The general framework of incorporating inherent anisotropy based on the microstructure within the hyperelasticity has been recently presented by Houlsby et al. [29] and validated with the experimental evidence by Amorosi et al. [2]. Another notable works on anisotropic hyperelastic models for soils have been reported in the literature by Gajo and Bigoni [17, 18] and Xiao et al. [54]. An interesting method of incorporating inherent cross-anisotropy into hyperelastic model by scaling of stiffness has been shown by Niemunis et al. [44].

\section{Formulation of the model}

In the elasto-plastic modelling, the initial stiffness is considered elastic within a small region in the stress or strain space. A constitutive model of elastic behaviour should provide a closed stress or strain loop within the elastic locus when material undergoes a closed strain or stress loop, respectively. Additionally, neither the dissipation nor the generation of the elastic energy is allowed in such process. It means that the following conditions must be fulfilled:

$$
\oint D_{i j k l}^{\mathrm{t}} \mathrm{d} \varepsilon_{k l}^{\mathrm{e}}=0, \quad \oint C_{i j k l}^{\mathrm{t}} \mathrm{d} \sigma_{k l}=0, \quad \oint \sigma_{i j} \mathrm{~d} \varepsilon_{i j}^{\mathrm{e}}=0,
$$

where $\mathbf{C}^{\mathrm{t}}$ and $\mathbf{D}^{\mathrm{t}}$ are the fourth-order tangent elastic compliance and stiffness tensors, respectively. If the thermodynamic requirements from Eq. 8 are satisfied, the model is considered hyperelastic. The most convenient way to formulate a truly conservative hyperelastic model is to derive it from the elastic potential function $W[25,28,43]$. It is a scalar function of stress-negative Gibbs free energy $W(\boldsymbol{\sigma})$ or elastic strain-Helmholtz free energy $W\left(\boldsymbol{\varepsilon}^{\mathrm{e}}\right)$. An elastic potential function is not a subject to any direct measurements; hence, it should be elaborated, for example, by inspection and analysis of its derivatives constituting the compliance or stiffness operators. In the case of first stress derivative of $W(\boldsymbol{\sigma})$, we obtain a secant compliance tensor $\mathrm{C}^{\mathrm{S}}$ relating the elastic strain and stress:

$\varepsilon_{i j}^{\mathrm{e}}=\frac{\partial W(\boldsymbol{\sigma})}{\partial \sigma_{i j}}=C_{i j k l}^{\mathrm{s}} \sigma_{k l}$.

To obtain a tangent compliance tensor $\mathbf{C}^{\mathrm{t}}$ that relates the elastic strain and stress rates, the second stress derivative of $W(\boldsymbol{\sigma})$ should be calculated:

$\dot{\varepsilon}_{i j}^{\mathrm{e}}=C_{i j k l}^{\mathrm{t}} \dot{\sigma}_{k l}=\frac{\partial^{2} W(\boldsymbol{\sigma})}{\partial \sigma_{i j} \partial \sigma_{k l}} \dot{\sigma}_{k l}$.

If an elastic potential is expressed as a function of elastic strain $W\left(\varepsilon^{\mathrm{e}}\right)$, we obtain secant and tangent stiffness tensors, $\mathbf{D}^{\mathrm{S}}$ and $\mathbf{D}^{\mathrm{t}}$, by analogous differentiations with respect to the elastic strain. 
A comparison of different stiffness formulations and investigation of the influence of their parameters may be achieved by confronting the so-called response envelopes [22]. Response envelope is a polar diagram of the tangent stiffness or compliance tensor. It is usually shown in the triaxial stress or strain plane $\left(\sqrt{2} \sigma_{3}-\sigma_{1}, \sqrt{2} \varepsilon_{3}^{\mathrm{e}}-\varepsilon_{1}^{\mathrm{e}}\right)$ as a closed curve representing the stiffness or compliance response to a circular stress or strain probe, respectively. Schematic description of obtaining the stiffness response envelope due to strain probe is shown in Fig. 2. Response envelopes can be obtained also experimentally. However, it is a complicated technique that still remains under development. Experimental response envelopes for sands have been reported by Danne and Hettler [13] and Knittel et al. [33].

\subsection{Basic hyperelastic model with stress-induced anisotropy}

Among different proposals of elastic potential functions available in the literature, we decided to make use of the stress-dependent formulation by Vermeer [51]. This function will be later modified and extended to account for inherent anisotropy. In our experience, it is an optimal solution that combines the practical functionality and simplicity associated with a small number of material parameters. The elastic potential has the following form:

$W(\boldsymbol{\sigma})=\frac{3 p_{\mathrm{ref}}^{1-\beta}}{2 G_{0}^{\mathrm{ref}}(1+\beta)}\left(\frac{2}{3} Q\right)^{(1+\beta) / 2}$,

where $G_{0}^{\text {ref }}$ is the small strain shear modulus measured at the isotropic reference stress $p=p_{\text {ref }}, \beta$ is a material parameter controlling the order of stress dependency of stiffness, and $Q$ is the stress invariant defined as:

$Q=\frac{1}{2} \operatorname{tr} \sigma^{2}=\frac{1}{2} \sigma_{i j} \sigma_{i j}$
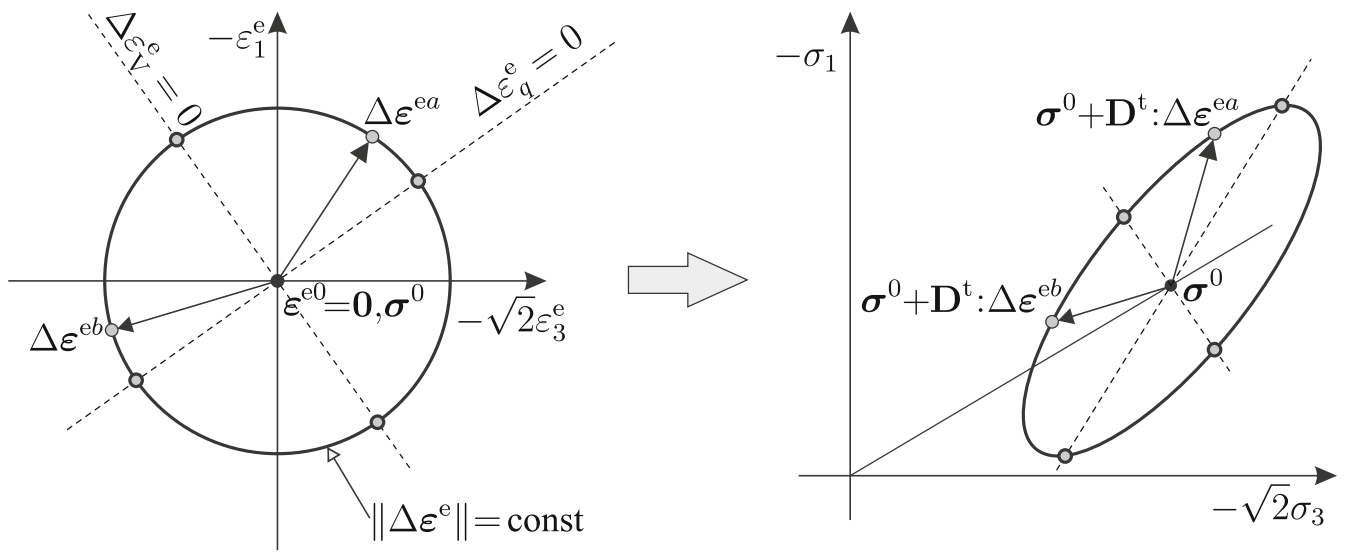

Fig. 2 Response envelope in the triaxial plane. Version with a circular strain probe and the ellipsoidal response in the triaxial stress plane $\left(\sigma_{2}=\sigma_{3}\right)$. Unlabelled dots correspond to purely deviatoric $\left(\Delta \varepsilon_{v}^{\mathrm{e}}=0\right)$ and purely volumetric $\left(\Delta \varepsilon_{q}^{\mathrm{e}}=0\right)$ strain increments 
or granular deposits, for which the values of $m$ are reported within the range from 0.3 to 0.7 , e.g. [4, 8, 14]. The coupling between $v$ and $m$ controlled by the value of $\beta$ is shown in Fig. 3. Additionally, response envelopes that illustrate the characteristic properties of the employed hyperelastic stiffness are presented. Size of the envelopes increases with the stress level which indicates the barotropy. Stress-induced anisotropy is evident by rotation of the envelopes resulting from the stress obliquity, i.e. $K \neq 1$.0. Elongation of the response envelopes, which shows a higher stiffness in compression along the stress paths $K=$ const, is directly controlled by the values of $\beta$. This effect may be compared to influence of the Poisson's ratio on stiffness in the isotropic Hooke's law. In Fig. 3, response envelopes of the hyperelastic stiffness are equivalent to those of the isotropic Hooke's law only for $p=p_{\text {ref }}$ and $K=1.0$ with the shear modulus and Poisson's ratio calculated with Eqs. 14 and 16, respectively.

\subsection{Definition of inherent cross-anisotropy}

To define a cross-anisotropic microstructure, we use second-order tensor $\mathbf{M}$ proposed in [7]. Its components are calculated from the following dyadic product:

$\mathbf{M}=\mathbf{v} \otimes \mathbf{v}, \quad M_{i j}=v_{i} v_{j}$,

where the unit vector $\mathbf{v}$ identifies the symmetry axis normal to the plane of isotropy. Using spherical coordinates $\phi, \theta$ in the geometrical framework shown in Fig. 4, the Cartesian coordinates of $\mathbf{v}$ are:

$\mathbf{v}=[\cos \theta, \sin \theta \cos \phi, \sin \theta \sin \phi]^{T}$.

After operation (Eq. 17), the general form of tensor $\mathbf{M}$ representing the cross-anisotropic microstructure is as follows:

$\mathbf{M}=$

$\left[\begin{array}{ccc}\cos ^{2} \theta & \cos \theta \cos \phi \sin \theta & \cos \theta \sin \theta \sin \phi \\ \cos \theta \cos \phi \sin \theta & \cos ^{2} \phi \sin ^{2} \theta & \cos \phi \sin ^{2} \theta \sin \phi \\ \cos \theta \sin \theta \sin \phi & \cos \phi \sin ^{2} \theta \sin \phi & \sin ^{2} \theta \sin ^{2} \phi\end{array}\right]$.

In this paper, the horizontally oriented plane of isotropy with the vertical symmetry axis is usually assumed, i.e. $\mathbf{v}=[1,0,0]^{T}$ and $\theta=0$, which leads to:

$\mathbf{M}=\left[\begin{array}{lll}1 & 0 & 0 \\ 0 & 0 & 0 \\ 0 & 0 & 0\end{array}\right]$.

\subsubsection{Formulation based on the mixed stress- microstructure invariant}

The modelling aim is to incorporate information on the cross-anisotropic microstructure into the framework of hyperelasticity using tensor M. We start from the basic
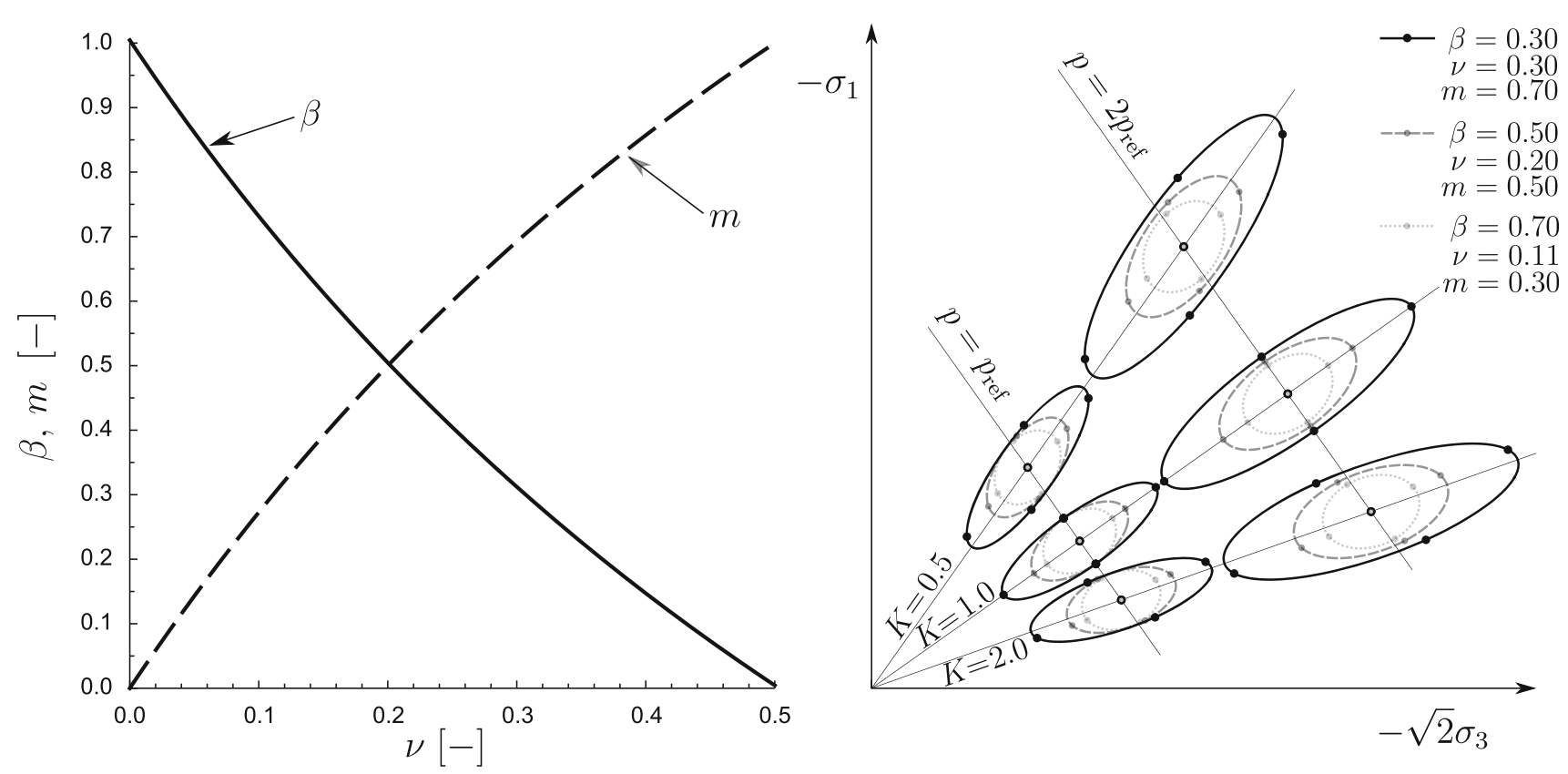

Fig. 3 Parameter $\beta$ coupling the order of stress dependency of stiffness $m$ and the Poisson's ratio $v$ as compared to the Hooke's elasticity at isotropic reference stress conditions. Left hand side: relation between $\beta, m$ and $v$. Right hand side: response envelopes of Vermeer's basic hyperelastic model [51] for different stress ratios $K$, stress levels $p$ and $\beta$ values. Response envelopes are scaled for visualisation purposes 


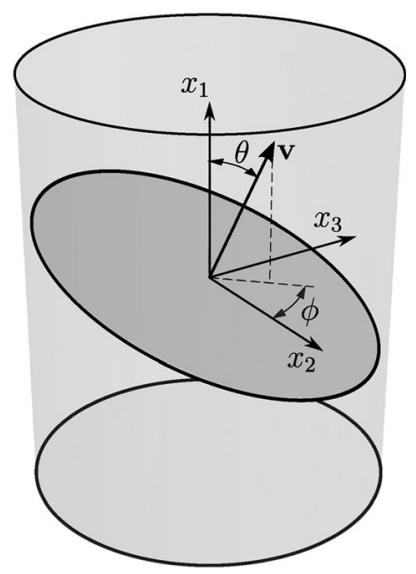

Fig. 4 Geometrical axes $x_{i}$, spherical coordinates $\phi, \theta$ and unit vector $\mathbf{v}$ defining the symmetry axis normal to the plane of isotropy as used in this paper

function used to derive the stiffness or compliance operators, i.e. the elastic potential function $W$. According to $[5,7]$, a general scalar function of two symmetric tensorial arguments, here $f(\boldsymbol{\sigma}, \mathbf{M})$, can be expressed as a function of the following three groups of scalar invariants:

$\operatorname{tr} \boldsymbol{\sigma}=\sigma_{i i}, \operatorname{tr} \boldsymbol{\sigma}^{2}=\sigma_{i j} \sigma_{i j}, \operatorname{tr} \boldsymbol{\sigma}^{3}=\sigma_{i j} \sigma_{j k} \sigma_{k i}$,

$\operatorname{tr} \mathbf{M}, \operatorname{tr} \mathbf{M}^{2}, \operatorname{tr} \mathbf{M}^{3}$

$\operatorname{tr}(\boldsymbol{\sigma} \cdot \mathbf{M}), \operatorname{tr}\left(\boldsymbol{\sigma}^{2} \cdot \mathbf{M}\right), \operatorname{tr}\left(\boldsymbol{\sigma} \cdot \mathbf{M}^{2}\right), \operatorname{tr}\left(\boldsymbol{\sigma}^{2} \cdot \mathbf{M}^{2}\right)$.

They represent the stress, microstructure and jointed stress-microstructure invariants, respectively. In the general case of cross-anisotropy (Eq. 19), all three invariants of microstructure in Eq. 22 are equal to unity and $\mathbf{M}=\mathbf{M}^{2}$. Hence, influence of the microstructure is significant only in the case of jointed invariants $\operatorname{tr}(\boldsymbol{\sigma} \cdot \mathbf{M})$ and $\operatorname{tr}\left(\boldsymbol{\sigma}^{2} \cdot \mathbf{M}\right)$. To denote functions of both stress and jointed invariants, we apply the overline symbol $\overline{()}$. An elastic potential function may be expressed in the following form:

$$
\begin{aligned}
& \bar{W}(\boldsymbol{\sigma}, \mathbf{M})=\bar{W}\left(\left[\xi_{1} \operatorname{tr} \boldsymbol{\sigma}+\xi_{2} \operatorname{tr}(\boldsymbol{\sigma} \cdot \mathbf{M})\right],\right. \\
& \left.\left[\xi_{3} \operatorname{tr} \boldsymbol{\sigma}^{2}+\xi_{4} \operatorname{tr}\left(\boldsymbol{\sigma}^{2} \cdot \mathbf{M}\right)\right], \operatorname{tr} \boldsymbol{\sigma}^{3}\right),
\end{aligned}
$$

with parameters $\xi_{i}$ as the additional scalar multipliers.

The basic elastic potential without inherent cross-anisotropy in Eq. 11 is a function of only one stress invariant $Q$. An analogous joint stress-microstructure invariant $Q_{M}$ may be defined as:

$Q_{M}=\frac{1}{2} \operatorname{tr}\left(\boldsymbol{\sigma}^{2} \cdot \mathbf{M}\right)=\frac{1}{2} M_{a b} \sigma_{b c} \sigma_{c a}$

As in Eq. 24, we can group the invariants $Q$ and $Q_{M}$ to obtain the following mixed invariant:

$$
\begin{aligned}
\bar{Q}= & c_{1} Q+c_{2} Q_{M}=\frac{1}{2} \overbrace{\left(c_{1} \delta_{a b}+c_{2} M_{a b}\right)}^{m_{a b}} \sigma_{b c} \sigma_{c a} \\
= & \frac{1}{2} m_{a b} \sigma_{b c} \sigma_{c a}
\end{aligned}
$$

where $c_{1}$ and $c_{2}$ are the new material constants. Finally, the incorporation of inherent cross-anisotropy into the elastic potential is attained by simply replacing the stress invariant $Q$ in Eq. 11 with its mixed equivalent $\bar{Q}$ :

$\bar{W}(\boldsymbol{\sigma}, \mathbf{M})=\frac{3 p_{\text {ref }}^{1-\beta}}{2 G_{0}^{\text {ref }}(1+\beta)}\left(\frac{2}{3} \bar{Q}\right)^{(1+\beta) / 2}$.

Constant $c_{1}$ controls the intensity of pure stress dependency of stiffness. Constant $c_{2}$ introduces inherent cross-anisotropic stiffness component with a fixed orientation of the symmetry axis, collinear with vector $\mathbf{v}$. In the case of $c_{1}=$ 1.0 and $c_{2}=0.0$, inherent cross-anisotropic component is deactivated and the basic potential function from Eq. 11 is recovered. As a rule, when $c_{1}$ is kept constant and $c_{2}>0.0$, inherent stiffness in the plane of isotropy is higher than in the direction of symmetry axis, i.e. $\alpha_{G}, \alpha_{E}>1.0$. Otherwise, when $c_{1}=$ const and $c_{2}<0.0$, inherent cross-anisotropy coefficients are $\alpha_{G}, \alpha_{E}<1.0$. Practically, the influence of parameter $c_{1}$ is a proportional scaling of the stiffness. The influence of $c_{1}$ on the directional distribution of stiffness is minor and its intensity depends on the current value of $c_{2}$. In order to simplify the presentation of the new model, we prefer to keep $c_{1}=1.0$ in the simulations and to control the directional properties of stiffness only by parameter $c_{2}$. We address the problem of parameter identification later in this paper.

In derivation of the secant hyperelastic strain-stress relation and the tangent compliance tensor, we need the partial derivative of the mixed invariant $\bar{Q}$ :

$\frac{\partial \bar{Q}}{\partial \sigma_{i j}}=\frac{1}{2}\left(\sigma_{a j} m_{a i}+\sigma_{b i} m_{b j}\right)$.

The secant strain-stress relation is obtained as:

$\varepsilon_{i j}^{\mathrm{e}}=\frac{\partial \bar{W}(\boldsymbol{\sigma}, \mathbf{M})}{\partial \sigma_{i j}}=\frac{1}{4 \bar{G}_{0}}\left(\sigma_{a j} m_{a i}+\sigma_{b i} m_{b j}\right)$

with

$\bar{G}_{0}=G_{0}^{\mathrm{ref}}\left(\frac{\sqrt{\frac{2}{3} \bar{Q}}}{p_{\text {ref }}}\right)^{1-\beta}$

The influence of $c_{2}$ value controlling inherent cross-anisotropic stiffness component may be observed by a qualitative analysis of plots showing the elastic strain distribution from Eq. 29. It is presented in Fig. 5. The influence of $c_{2}$ value is clear when comparing stress 


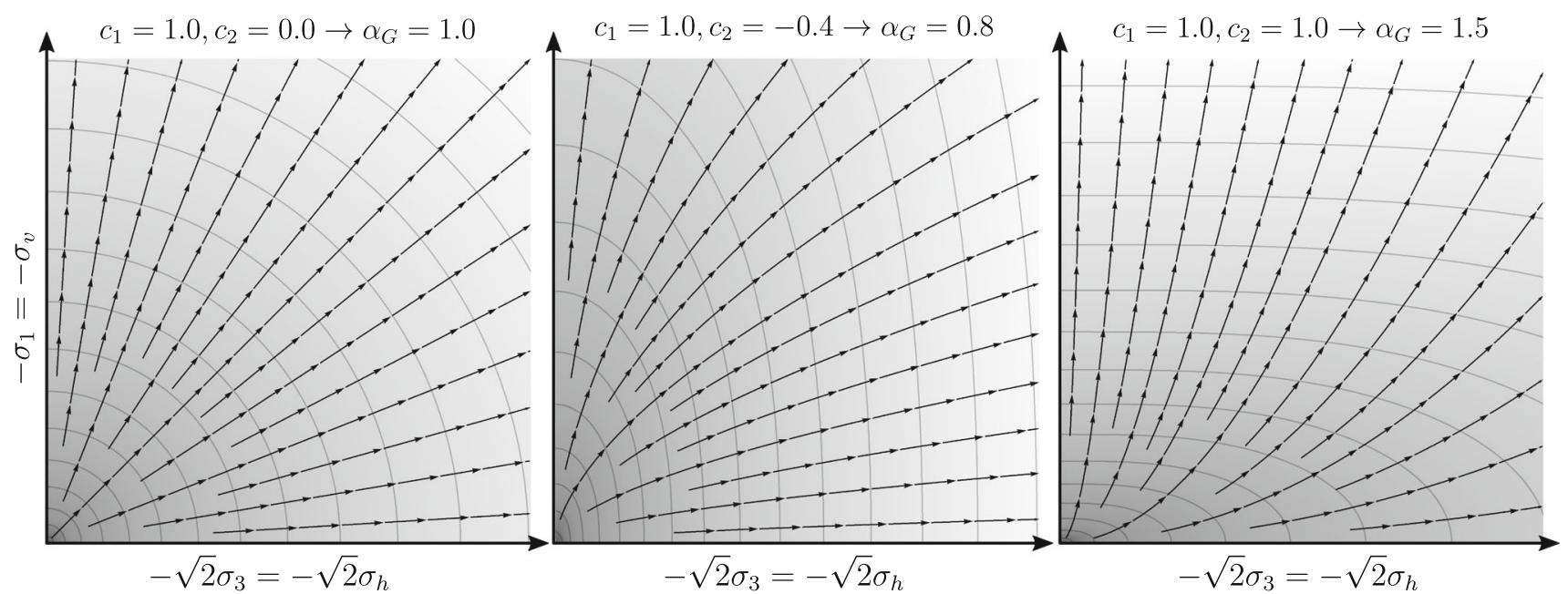

Fig. 5 Elastic strain distribution in the triaxial stress plane resulting from the secant relation (Eq. 29). Vector streams depict directions of $\left[\partial \bar{W} / \partial \sigma_{h}, \partial \bar{W} / \partial \sigma_{v}\right]^{T}$ and isolines identify the constant values of $\|\partial \bar{W} / \partial \boldsymbol{\sigma}\|$. In all the plots, values of $c_{1}$ and the reference shear modulus in vertical plane $G_{v h}^{\text {ref }}$ are kept constant

distances between the subsequent isolines of $\|\partial \bar{W} / \partial \boldsymbol{\sigma}\|$ along different vector streams. These distances are proportional to the directional stiffness. In the left plot, the inherent cross-anisotropic component is absent as $c_{2}=0.0$ and the isolines form circles. The distribution changes in the middle $\left(c_{2}=-0.4\right)$ and right $\left(c_{2}=1.0\right)$ plots, leading to the higher stiffness in the vertical and horizontal directions, respectively.

Another important feature of the proposed model, which can be illustrated on the basis of secant strain-stress relation (Eq. 29), is the shape of the constant volumetric strain $\varepsilon_{\mathrm{V}}^{\mathrm{e}}$ and the shear strain $\varepsilon_{q}^{\mathrm{e}}$ contours. They are usually presented in the $p-q$ triaxial plane, e.g. [2, 28]. Such volumetric and shear strain contours for the proposed model are presented in Fig. 6.
It should be noted that $\varepsilon_{\mathrm{V}}^{\mathrm{e}}=$ const contours correspond to the undrained triaxial compression stress paths for elastic behaviour. Hence, the imposed inherent cross-anisotropy using parameter $c_{2}$ influences direction of the undrained stress paths. Consequently, if the proposed hyperelastic description is a part of an elasto-plastic model, it will impact the undrained shear strength.

Finally, the tangent compliance of the hyperelastic model with stress-induced anisotropy and inherent crossanisotropy is obtained from the following differentiation:

$$
C_{i j k l}^{\mathrm{t}}=\frac{\partial^{2} \bar{W}(\boldsymbol{\sigma}, \mathbf{M})}{\partial \sigma_{i j} \partial \sigma_{k l}}=\frac{1}{4 \bar{G}_{0}} A_{i j k l},
$$

where
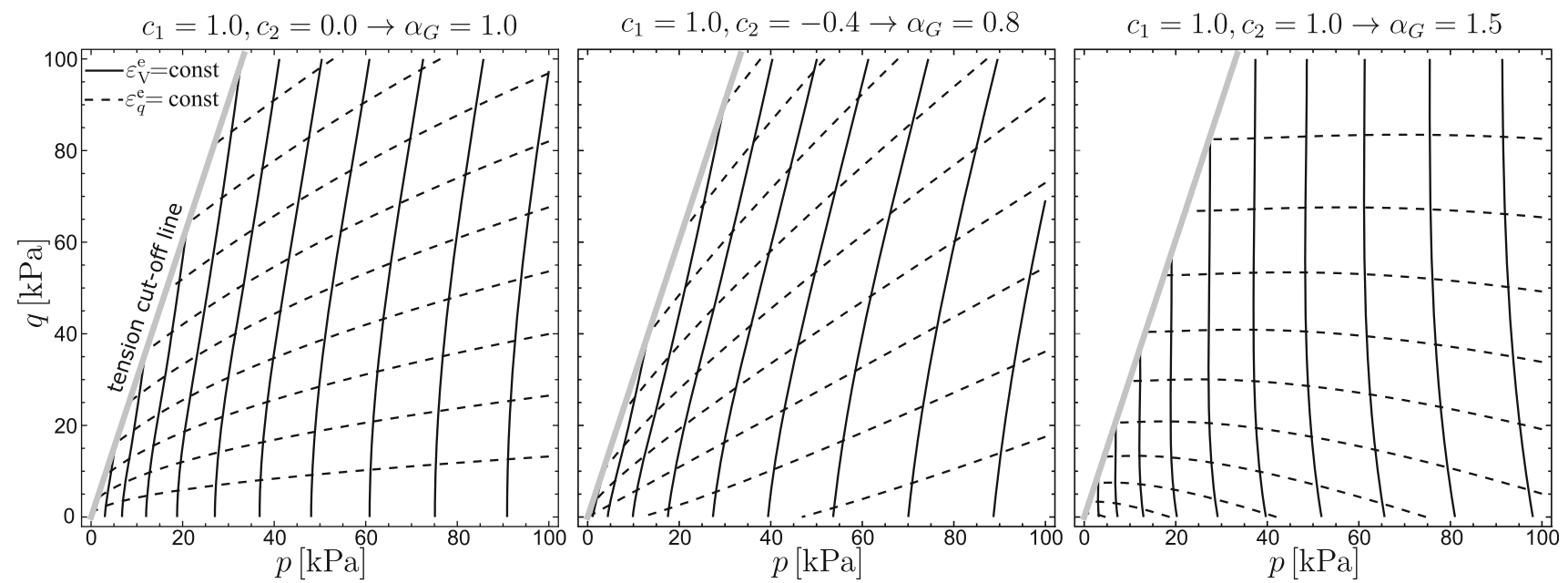

Fig. 6 Contours of the constant volumetric $\varepsilon_{\mathrm{V}}^{\mathrm{e}}=\varepsilon_{1}^{\mathrm{e}}+2 \varepsilon_{3}^{\mathrm{e}}$ and shear $\varepsilon_{q}^{\mathrm{e}}=2 / 3\left(\varepsilon_{1}^{\mathrm{e}}-\varepsilon_{3}^{\mathrm{e}}\right)$ elastic strains in triaxial $p-q$ plane for different values of $c_{2}$. The same set of parameters as in Fig. 5 


$$
\begin{aligned}
& A_{i j k l}=\left(\delta_{j l} m_{i k}+\delta_{i l} m_{j k}\right)^{\mathrm{symm}} \\
& -(1-\beta) \frac{\left(\sigma_{a l} m_{a k}+\sigma_{b k} m_{b l}\right)\left(\sigma_{a j} m_{a i}+\sigma_{b i} m_{b j}\right)}{4 \bar{Q}},
\end{aligned}
$$

and

$$
\left(\delta_{j l} m_{i k}+\delta_{i l} m_{j k}\right)^{\mathrm{symm}}=\frac{1}{2}\left(\delta_{j l} m_{i k}+\delta_{j k} m_{i l}+\delta_{i l} m_{j k}+\delta_{i k} m_{j l}\right) .
$$

The tangent stiffness matrix can be derived analytically or numerically by inversion of the tangent compliance tensor in the Voigt notation. For details of such operations, the reader is referred to [41]. It should be noted that the derivation of hyperelastic stiffness via the inversion of compliance matrix, applied in numerical calculations presented in this paper, is an alternative way to the classic and more elegant method presented in, for example, [29] or [44]. Elastic potential expressed as a function of elastic strain may be obtained from stress functions (11) or (27) by Legendre transform. Finally, the hyperelastic stiffness may be obtained directly from differentiation of the transformed potential with respect to the elastic strain.

\section{Features of the model and material constants}

\subsection{Inherent cross-anisotropy at isotropic stress conditions}

For practical purposes, it is important to relate material constants of the proposed model, i.e. $G_{0}^{\text {ref }}, \beta, c_{1}, c_{2}, p_{\text {ref }}$ to parameters measured in the geotechnical laboratories or reported in the literature. The inherent anisotropic elastic parameters can only be determined in an isotropic stress state $\boldsymbol{\sigma}=-p \boldsymbol{\delta}$. With $p=p_{\text {ref }}$, the measured elastic moduli are indicated as reference and consequently superscript ()$^{\text {ref }}$ is used. Applying vertically and horizontally oriented pairs of bender elements on a triaxial sample allows determination of shear moduli $G_{v h}, G_{h h}$ and calculation of the corresponding anisotropy coefficient $\alpha_{G}$, e.g. $[15,20]$. Additionally, the vertical Young's modulus $E_{v}$ can be measured, if the triaxial device is equipped with a high accuracy axial strain transducer. Determination of the horizontal Young's modulus $E_{h}$ requires incorporation of some more sophisticated procedures, such as testing horizontally trimmed samples or investigations in the true triaxial device. The reference shear modulus $G_{0}^{\text {ref }}$, being the basic stiffness parameter in the model, represents the value of $G_{v h}^{\text {ref }}$ only if the material is inherently isotropic, i.e. $c_{2}=0.0$ which implies $\alpha_{G}=1.0$. In inherently cross- anisotropic soils, the value of $G_{0}^{\text {ref }}$ should be estimated on the basis of directly measured $G_{v h}^{\text {ref }}$ or $E_{v}^{\text {ref }}$ and anisotropy coefficient $\alpha_{G}$ or $\alpha_{E}$. To obtain the relations for shear moduli, $G_{v h}$ and $G_{h h}$, we need to inspect the response of the proposed anisotropic model to the following stress increments:

$\Delta \boldsymbol{\sigma}=\left[\begin{array}{ccc}0 & \Delta \sigma_{v h} & 0 \\ \Delta \sigma_{v h} & 0 & 0 \\ 0 & 0 & 0\end{array}\right], \quad\left[\begin{array}{ccc}0 & 0 & 0 \\ 0 & 0 & \Delta \sigma_{h h} \\ 0 & \Delta \sigma_{h h} & 0\end{array}\right]$,

respectively. In the case of Young's moduli, $E_{v}$ and $E_{h}$, we examine the response to:

$\Delta \boldsymbol{\sigma}=\left[\begin{array}{ccc}\Delta \sigma_{v} & 0 & 0 \\ 0 & 0 & 0 \\ 0 & 0 & 0\end{array}\right], \quad\left[\begin{array}{ccc}0 & 0 & 0 \\ 0 & \Delta \sigma_{h} & 0 \\ 0 & 0 & 0\end{array}\right]$,

respectively. The initial isotropic stress condition is $\sigma^{0}=$ $-p_{0} \boldsymbol{\delta}$ and the cross-anisotropic microstructure is represented by tensor $\mathbf{M}$ as defined in Eq. 20. Components of the incremental elastic strain response to the stress increments Eqs. 34 and 35 are calculated using tangent compliance tensor (Eq. 31):

$\Delta \varepsilon^{\mathrm{e}}=\mathbf{C}^{\mathrm{t}}\left(\boldsymbol{\sigma}^{0}, \mathbf{M}\right): \Delta \boldsymbol{\sigma}$.

The following formulas for the inherent cross-anisotropic stiffness moduli are obtained:

$G_{v h}=\frac{\Delta \sigma_{v h}}{2 \Delta \varepsilon_{12}^{\mathrm{e}}}=G_{0}^{\mathrm{ref}} \frac{\left(\frac{p_{0}}{p_{\text {ref }}} \sqrt{c_{1}+\frac{1}{3} c_{2}}\right)^{1-\beta}}{c_{1}+\frac{1}{2} c_{2}}$,
$G_{h h}=\frac{\Delta \sigma_{v h}}{2 \Delta \varepsilon_{23}^{\mathrm{e}}}=G_{0}^{\mathrm{ref}} \frac{\left(\frac{p_{0}}{p_{\text {ref }}} \sqrt{c_{1}+\frac{1}{3} c_{2}}\right)^{1-\beta}}{c_{1}}$,
$E_{v}=\frac{\Delta \sigma_{v}}{\Delta \varepsilon_{11}^{\mathrm{e}}}=2 G_{0}^{\mathrm{ref}} \frac{\left(3 c_{1}+c_{2}\right)\left(\frac{p_{0}}{p_{\text {ref }}} \sqrt{c_{1}+\frac{1}{3} c_{2}}\right)^{1-\beta}}{\left(c_{1}+c_{2}\right)\left[c_{2} \beta+c_{1}(2+\beta)\right]}$,

$E_{h}=\frac{\Delta \sigma_{v}}{\Delta \varepsilon_{22}^{\mathrm{e}}}=2 G_{0}^{\mathrm{ref}} \frac{\left(3 c_{1}+c_{2}\right)\left(\frac{p_{0}}{p_{\text {ref }}} \sqrt{c_{1}+\frac{1}{3} c_{2}}\right)^{1-\beta}}{c_{1}\left[c_{2}+c_{1}(2+\beta)\right]}$.

Using the elastic strain responses to the stress increments (Eq. 35), we define the cross-anisotropic Poisson's ratios:

$v_{v h}=\frac{-\Delta \varepsilon_{22}^{\mathrm{e}}}{\Delta \varepsilon_{11}^{\mathrm{e}}}=\frac{c_{1}(1-\beta)}{c_{2} \beta+c_{1}(2+\beta)}$, 


$$
v_{h h}=\frac{-\Delta \varepsilon_{33}^{\mathrm{e}}}{\Delta \varepsilon_{22}^{\mathrm{e}}}=\frac{c_{1}(1-\beta)}{c_{2}+c_{1}(2+\beta)} .
$$

Now, the anisotropy coefficients (Eqs. 3-5) can be derived for the proposed model:

$$
\begin{aligned}
& \alpha_{E}=\frac{E_{h}}{E_{v}}=\frac{\left(c_{1}+c_{2}\right)\left[c_{2} \beta+c_{1}(2+\beta)\right]}{c_{1}\left[c_{2}+c_{1}(2+\beta)\right]}, \\
& \alpha_{G}=\frac{G_{h h}}{G_{v h}}=1+\frac{c_{2}}{2 c_{1}}, \\
& \alpha_{v}=\frac{v_{h h}}{v_{v h}}=\frac{c_{2} \beta+c_{1}(2+\beta)}{c_{2}+c_{1}(2+\beta)} .
\end{aligned}
$$

In practice, it is feasible to determine the following pairs of the cross-anisotropic parameters in triaxial tests at a reference isotropic stress state represented by $p_{0}=p_{\text {ref }}$ :

- $G_{v h}^{\mathrm{ref}}, \alpha_{G}$, using vertical and horizontal pairs of bender elements;

- $E_{v}^{\text {ref }}, \alpha_{G}$, using bender elements as above in drained compression with high accuracy axial strain measurements;

- $E_{\mathrm{uv}}^{\mathrm{ref}}, \alpha_{G}$, using bender elements as above in undrained compression with high accuracy axial strain measurements.

In order to simplify estimation of the model parameters, it is proposed to fix the constant value of $c_{1}=1.0$ and to adjust $c_{2}$ with $\alpha_{G}$ using Eq. 44:

$c_{2}=2\left(\alpha_{G}-1\right)$.

Having $G_{v h}^{\mathrm{ref}}$ or $E_{v}^{\mathrm{ref}}$ and $\alpha_{G}$ determined, the reference shear modulus $G_{0}^{\text {ref }}$ can be calculated from the following equations:

$$
\begin{aligned}
& G_{0}^{\mathrm{ref}}\left(G_{v h}^{\mathrm{ref}}, \alpha_{G}, \beta\right)=G_{v h}^{\mathrm{ref}} \alpha_{G}\left(\sqrt{\frac{1+2 \alpha_{G}}{3}}\right)^{\beta-1}, \\
& G_{0}^{\mathrm{ref}}\left(E_{v}^{\mathrm{ref}}, \alpha_{G}, \beta\right) \\
& =E_{v}^{\mathrm{ref}} \frac{1-2 \alpha_{G}}{1+2 \alpha_{G}}\left(\frac{1-2 \alpha_{G}}{2} \beta-1\right)\left(\sqrt{\frac{1+2 \alpha_{G}}{3}}\right)^{\beta-1} .
\end{aligned}
$$

In undrained triaxial compression tests, the small strain undrained Young's modulus in direction of the symmetry axis $E_{\mathrm{uv}}^{\mathrm{ref}}$ can be measured [20]. In order to derive $E_{\mathrm{uv}}^{\mathrm{ref}}$, we have to inspect the model response to the following elastic strain increment:

$\Delta \varepsilon^{\mathrm{e}}=\left[\begin{array}{ccc}\Delta \varepsilon_{v}^{\mathrm{e}} & 0 & 0 \\ 0 & -\Delta \varepsilon_{v}^{\mathrm{e}} / 2 & 0 \\ 0 & 0 & -\Delta \varepsilon_{v}^{\mathrm{e}} / 2\end{array}\right]$
Components of the incremental elastic stress response under isotropic stress condition $\boldsymbol{\sigma}^{0}=-p_{0} \boldsymbol{\delta}$ are calculated using the tangent stiffness tensor:

$\Delta \boldsymbol{\sigma}=\mathbf{D}^{\mathrm{t}}\left(\boldsymbol{\sigma}^{0}, \mathbf{M}\right): \Delta \boldsymbol{\varepsilon}^{\mathrm{e}}$,

and the undrained Young's modulus can be calculated from:

$E_{\mathrm{uv}}=\frac{\Delta \sigma_{11}-\Delta \sigma_{22}}{\Delta \varepsilon_{v}^{\mathrm{e}}}=G_{0}^{\mathrm{ref}} \frac{\left(3 c_{1}+c_{2}\right)\left(\frac{p_{0}}{p_{\text {ref }}} \sqrt{c_{1}+\frac{1}{3} c_{2}}\right)^{1-\beta}}{c_{1}\left(c_{1}+c_{2}\right)}$.

After inversion of the above equation, assuming $p_{0}=p_{\text {ref }}$, $c_{1}=1.0$ and $c_{2}$ according to Eq. 46, the reference shear modulus $G_{0}^{\text {ref }}$ can be obtained as follows:

$$
G_{0}^{\mathrm{ref}}\left(E_{\mathrm{uv}}^{\mathrm{ref}}, \alpha_{G}, \beta\right)=E_{\mathrm{uv}}^{\mathrm{ref}} \frac{-1+2 \alpha_{G}}{1+2 \alpha_{G}}\left(\sqrt{\frac{1+2 \alpha_{G}}{3}}\right)^{\beta-1}
$$

\subsection{Relations between inherent anisotropy coefficients}

Combining the relations for anisotropy coefficients (Eqs. 43-45), it is possible to define $\alpha_{E}$ and $\alpha_{v}$ as functions of parameters $\alpha_{G}$ and $\beta$ :

$$
\begin{aligned}
& \alpha_{E}\left(\alpha_{G}, \beta\right)=\frac{\left(2 \alpha_{G}-1\right)\left[2+\left(2 \alpha_{G}-1\right) \beta\right]}{2 \alpha_{G}+\beta}, \\
& \alpha_{v}\left(\alpha_{G}, \beta\right)=\frac{\left(2 \alpha_{G}-1\right) \beta+2}{2 \alpha_{G}+\beta} .
\end{aligned}
$$

The obtained relations, unlike in Eqs. 6 and 7, are not exponential and the corresponding anisotropy exponents $x_{G E}$ and $x_{G v}$ depend on the values of $\alpha_{G}$ and $\beta$. In Fig. 7, a graphical comparison of relations (Eqs. 53, 54) with exponential functions is presented for $\beta=0.5$, which implies order of stress dependency $m=0.5$ often assumed in overconsolidated clays and sands. Another graphical comparison concerns relations between anisotropy coefficients for different values of $\beta$ as presented in Fig. 8. Since $\alpha_{G}$ is independent of $\beta$, we have examined changes of $\alpha_{E}$ and $\alpha_{v}$ with $\beta$ for two opposite cases of inherent crossanisotropy, i.e. higher stiffness along the symmetry axis $\left(\alpha_{G}=0.6\right)$ and higher stiffness in the plane of isotropy $\left(\alpha_{G}=2.0\right)$. Keeping the same range of $\beta$, we have also shown changes of exponent $x_{G E}$ for seven selected values of $\alpha_{G}$ between 0.6 and 3.0 as presented in Fig. 9 . 

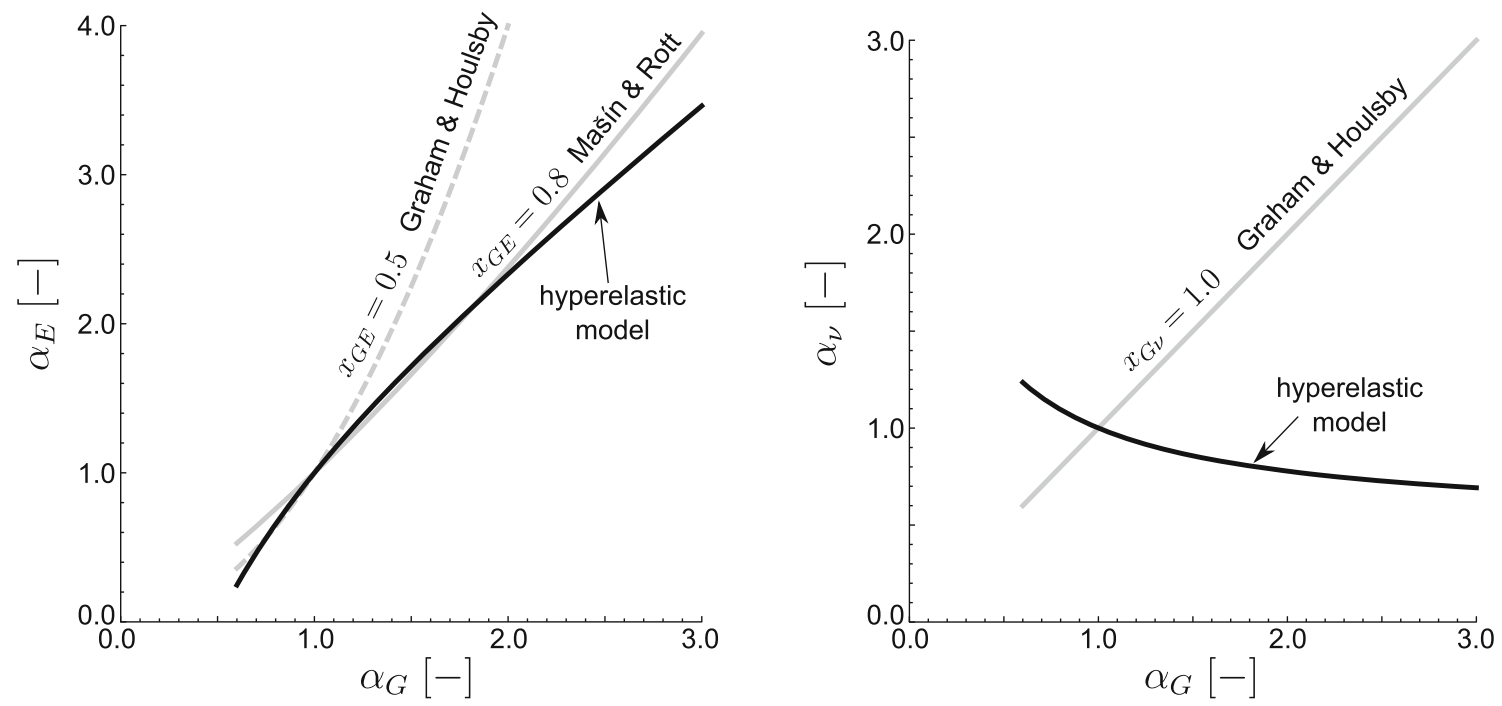

Fig. 7 Relations between anisotropy coefficients $\alpha_{E}, \alpha_{v}$ and $\alpha_{G}$ reproduced by the model for $\beta=0.5$. In the case of relation $\alpha_{E}\left(\alpha_{G}\right)$, it is close to findings presented by Mašín and Rott [38]. The relation $\alpha_{v}\left(\alpha_{G}\right)$ deviates from $\alpha_{v}=\alpha_{G}$ as assumed in the cross-anisotropic model by Graham and Houlsby [21]. However, experimental evidence in this case is still very scattered [38]
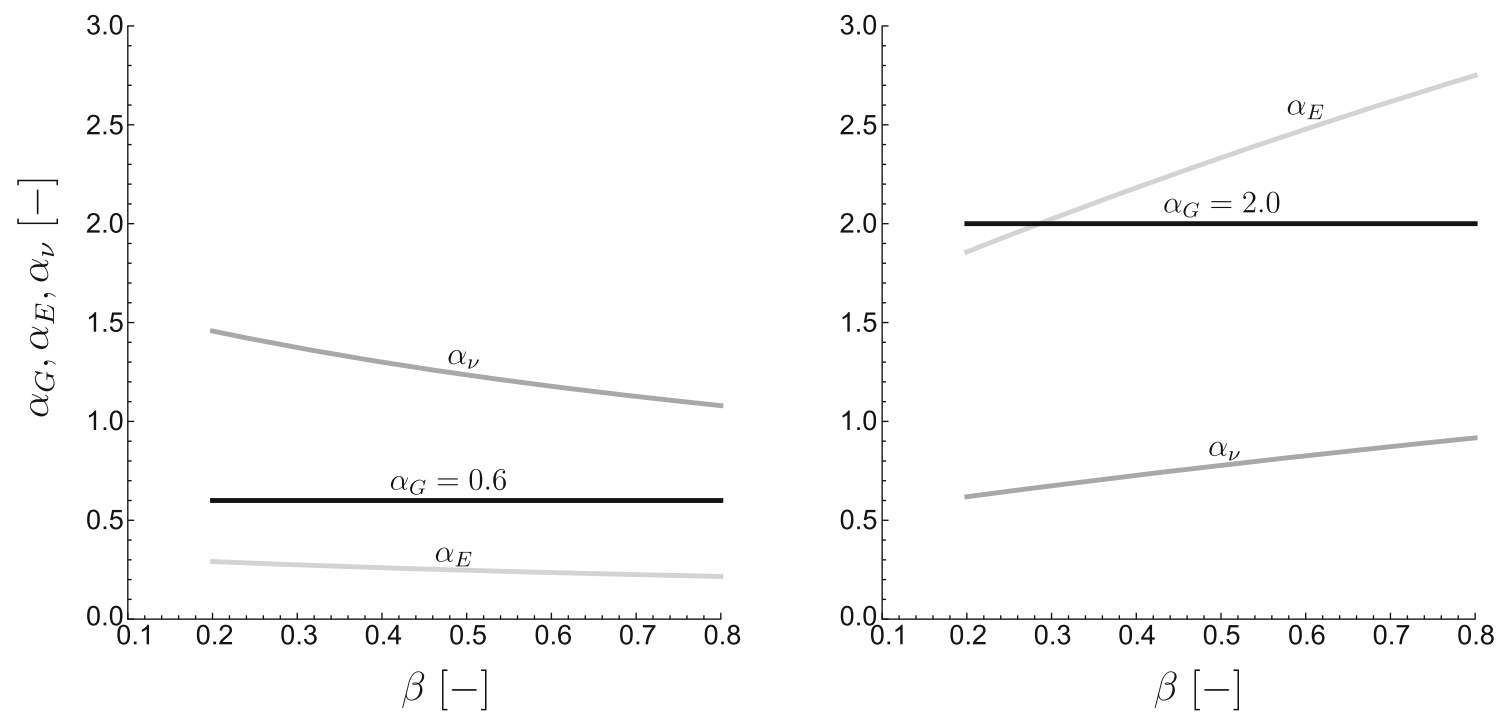

Fig. 8 Relations between anisotropy coefficients $\alpha_{E}, \alpha_{v}$ and $\alpha_{G}$ reproduced by the model for two opposite cases of inherent cross-anisotropy $\left(\alpha_{G}=0.6\right.$ and $\left.\alpha_{G}=2.0\right)$ and different values of parameter $\beta$

\subsubsection{Mixed anisotropy at axisymmetric and true triaxial stress conditions}

Changes of stiffness resulting from the superposition of variable stress-induced anisotropy and constant inherent cross-anisotropy may be illustrated qualitatively by comparison of response envelopes elaborated for different initial stress ratios $K$ and selected values of anisotropy coefficient $\alpha_{G}$. Such response envelopes at axisymmetric stress conditions are presented in Fig. 10. In the comparison, the simplified parameter set, i.e. $G_{v h}^{\text {ref }}, \alpha_{G}, \beta, p_{\text {ref }}$ is used. All parameters are kept constant and the influence of two inherent cross-anisotropy cases $\left(\alpha_{G}=0.7\right.$ and $\left.\alpha_{G}=2.0\right)$ is examined. The internal parameters, $G_{0}^{\text {ref }}$ and $c_{2}$, are calculated from Eqs. 47 and 46, respectively. Values of parameters $G_{v h}^{\text {ref }}$ and $p_{\text {ref }}$ scale solely the size of response envelopes. Hence, these values are chosen regarding only the visualisation purposes. Response envelopes presenting mixed stress-induced and inherent anisotropy are shown together with the reference envelopes without inherent anisotropy $\left(\alpha_{G}=1.0\right)$. All response envelopes are presented for mean stress level $p=p_{\text {ref }}$, three different stress ratios $K$ and $\beta=0.5$. It can be seen in Fig. 10 that for $\alpha_{G}<1.0$, the radial stress response to purely 


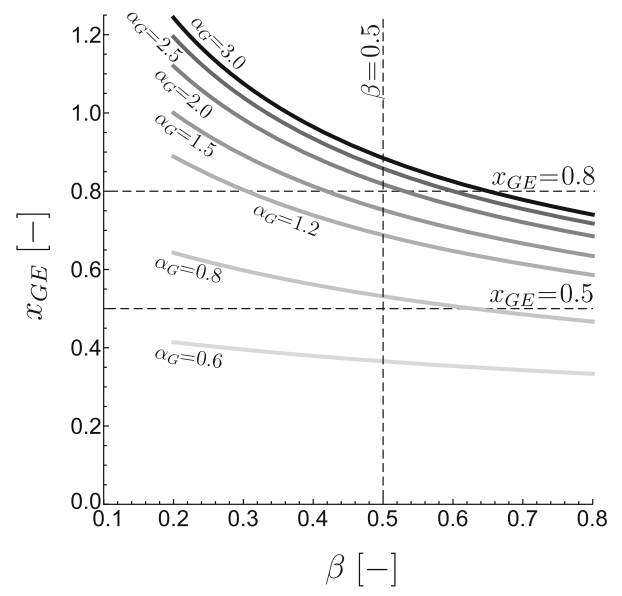

Fig. 9 Changes of anisotropy exponent $x_{G E}$ resulting from the model for different values of $\alpha_{G}$ and $\beta$

volumetric strain probes rotates anticlockwise and the radial stress response to purely deviatoric strain probes rotates clockwise relative to the response obtained for $\alpha_{G}=1.0$. As a result of such behaviour, the response envelopes seem to rotate anticlockwise, if $\alpha_{G}<1.0$. If $\alpha_{G}>1.0$, the rotations occur in the opposite direction.

Definitions of the anisotropy coefficients may be extended to account for axisymmetric stress conditions. This allows to investigate the mixed anisotropy quantitatively and to show how the stress obliquity $K$ influences the resulting stiffness. For this purpose, one needs to inspect the model response to stress increments (Eqs. 34, 35) starting from the following initial axisymmetric stress state:

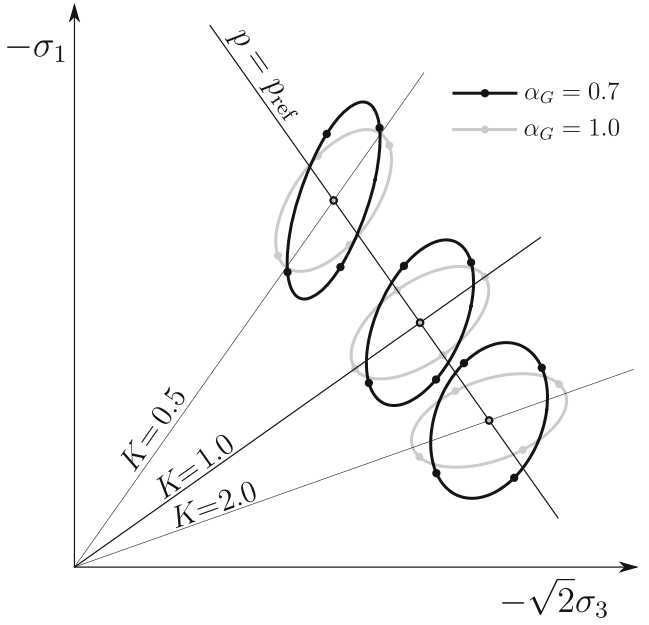

$$
\boldsymbol{\sigma}^{0}=\left[\begin{array}{ccc}
-\frac{3 p_{0}}{1+2 K} & 0 & 0 \\
0 & -\frac{3 K p_{0}}{1+2 K} & 0 \\
0 & 0 & -\frac{3 K p_{0}}{1+2 K}
\end{array}\right] .
$$

Using the internal model parameters, i.e. $G_{0}^{\mathrm{ref}}, c_{1}, c_{2}, \beta, p_{\text {ref }}$, in operation (Eq. 36), we obtain the same relation for anisotropy coefficient $\alpha_{G}$ as in Eq. 44. It is an important feature of the model that $\alpha_{G}$ is constant and independent of the stress obliquity at axisymmetric stress conditions. Analogously, it can be shown that $\alpha_{G}$ is also constant at a true triaxial stress state, i.e. for three different normal stress components and zero shear stress components. Hence, in the extended definitions of cross-anisotropic moduli. we use $\alpha_{G}$ assuming $c_{1}=1.0$ and (Eq. 46). The following formulas for the extended cross-anisotropic stiffness moduli are obtained:

$G_{v h}=\frac{G_{0}^{\mathrm{ref}}}{\alpha_{G}}\left(\frac{\frac{p_{0}}{p_{\text {ref }}} \sqrt{6 K^{2}+6 \alpha_{G}-3}}{1+2 K}\right)^{1-\beta}$,

$G_{h h}=\alpha_{G} G_{v h}$,

$E_{v}=2 G_{0}^{\mathrm{ref}}\left(\frac{\frac{p_{0}}{p_{\text {ref }}} \sqrt{6 K^{2}+6 \alpha_{G}-3}}{1+2 K}\right)^{1-\beta}$

$$
\frac{\left(2 K^{2}+2 \alpha_{G}-1\right)}{\left(2 \alpha_{G}-1\right)\left[2 K^{2}+\left(2 \alpha_{G}-1\right) \beta\right]},
$$

$E_{h}=\alpha_{E} E_{v}$,

where

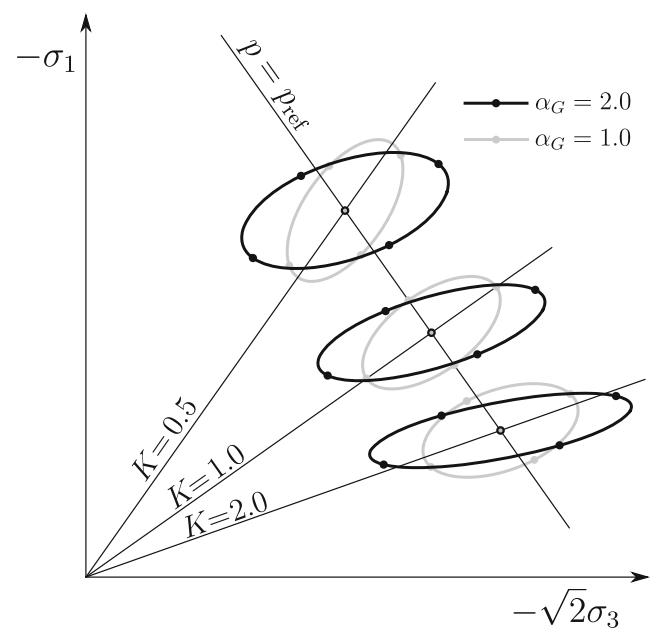

Fig. 10 Response envelopes of the proposed hyperelastic model. Envelopes are compared for the same mean stress level $p_{\text {ref }}$ and three different stress ratios $K$. Two values of the anisotropy coefficient are examined: $\alpha_{G}=0.7$ and $\alpha_{G}=2.0$. The grey response envelopes represent the basic hyperelastic model with stress-induced anisotropy only $\left(\alpha_{G}=1.0\right)$. They are drawn to facilitate comparisons 
$\alpha_{E}\left(\alpha_{G}, \beta, K\right)=\frac{\left(2 \alpha_{G}-1\right)\left[2 K^{2}+\left(2 \alpha_{G}-1\right) \beta\right]}{2 \alpha_{G}+K^{2}(1+\beta)-1}$.

Regarding the cross-anisotropic Poisson's ratios at axisymmetric stress conditions, the following formulas are obtained:

$v_{v h}=\frac{K(1-\beta)}{2 K^{2}+\left(2 \alpha_{G}-1\right) \beta}$,

$v_{h h}=\alpha_{v} v_{v h}$,

where

$\alpha_{v}\left(\alpha_{G}, \beta, K\right)=\frac{2 K^{3}+K\left(2 \alpha_{G}-1\right) \beta}{K^{2}(1+\beta)+2 \alpha_{G}-1}$.

The above extended definitions of anisotropy coefficients can be used to show changes of the mixed anisotropy with the stress ratio $K$ and coefficient $\alpha_{G}$ at axisymmetric stress states. This is presented for $\beta=0.5$ in Fig. 11. At the isotropic stress $(K=1.0)$, anisotropy coefficients represent the inherent values from Eq. 53 and 54. Deviation from the isotropic stress $(K \neq 1.0)$ results in a proportional increase or decrease in the value of anisotropy coefficients.

In real soils, the mixed anisotropy of initial stiffness may be more complex. All principal stress components may have different values with the principal axes rotated with respect to the geometrical axes. Moreover, the plane of isotropy may be non-horizontal. To visualise stiffness obtained with the model for such conditions, an orientation distribution function $\varrho^{-1 C}$ related to the fourth-order tangent compliance tensor can be used [52]:

$\varrho^{-1 C}\left(\mathbf{C}^{\mathrm{t}}, \mathbf{n}\right)=\left(n_{i} n_{j} C_{i j k l}^{\mathrm{t}} n_{k} n_{l}\right)^{-1}$.

It reflects a scalar value which is equal to the Young's modulus in the direction indicated by the unit vector $\mathbf{n}$.
After parametrisation of $\mathbf{n}$ in terms of spherical coordinates, as in Eq. 18, spherical plots of $\varrho^{-1 C}$ can be produced to illustrate the mixed anisotropy of the proposed hyperelastic stiffness. This is shown in Fig. 12 where cases (a)(d) present the growing complexity of stress and microstructural conditions in sequence. In Fig. 12a, both stress and microstructure are isotropic and Young's modulus distribution forms perfect sphere like in the isotropic Hooke's stiffness. In Fig. 12b, example true triaxial stress state is introduced; however, principal stress axes are collinear with the geometrical axes and the microstructure is still isotropic. The distribution forms ellipsoid reflecting stress-induced anisotropy from the basic hyperelastic Vermeer's stiffness in Eq. 15. The axis of symmetry $\mathbf{v}$ is shown vertical, but its orientation has no influence because $\alpha_{G}=1.0$. Inherent cross-anisotropy is introduced in Fig. $12 \mathrm{c}$ by imposing $\alpha_{G}=2.0$. This results in an increase of Young's modulus in all horizontal directions and the distribution diverges from a regular ellipsoid. Note that geometrical, microstructural and stress axes are still collinear and the plane of isotropy is horizontal. Rotation of the plane of isotropy representing the inherent microstructure is introduced in Fig. 12d. In all presented spherical plots, $G_{v h}^{\text {ref }}=$ const and $\beta=0.5$.

\section{Validation of the proposed model with experimental data}

The model has been validated with selected results of laboratory tests from the literature. The simplified set of model parameters, i.e. $G_{v h}^{\mathrm{ref}}, \alpha_{G}, \beta, p_{\text {ref }}$, is used. The obtained results are presented for sands and clays separately. In all referred element tests, the plane of isotropy in
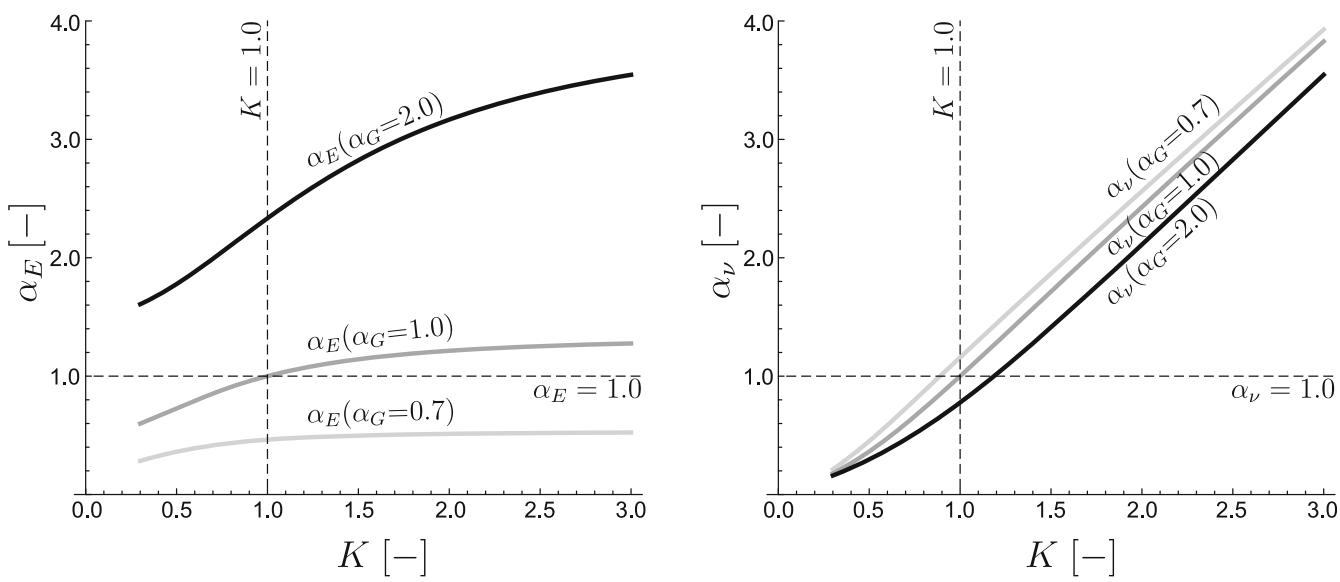

Fig. 11 Changes of anisotropy coefficients $\alpha_{E}$ and $\alpha_{v}$ with the stress ratio $K$ at axisymmetric stress conditions 


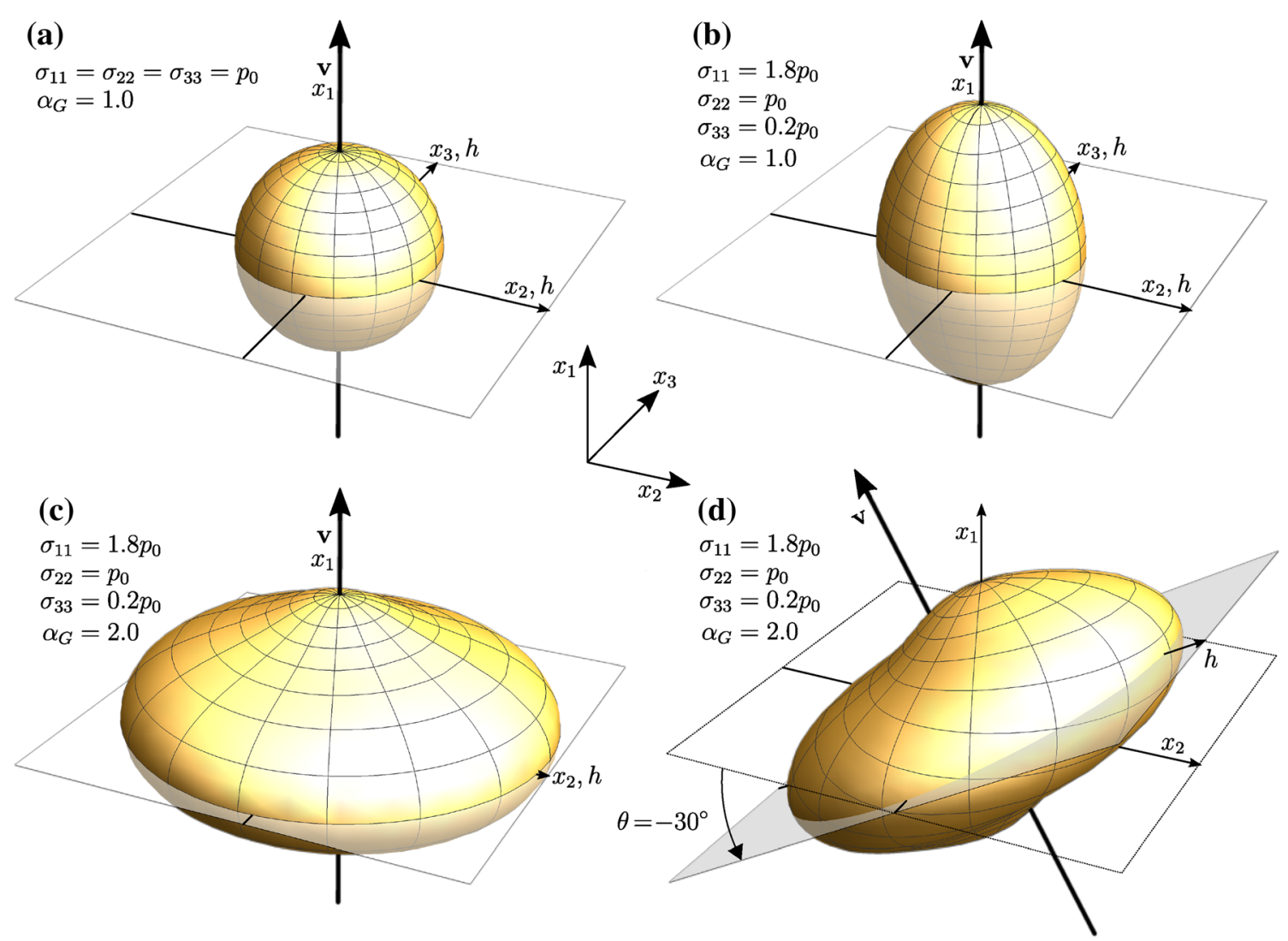

Fig. 12 Spherical plots illustrating three-dimensional polar distribution of stiffness reproduced by the model at different conditions: a fully isotropic case, $\mathbf{b}$ anisotropy induced by example true triaxial stress state $\left(\sigma_{i j}=0\right.$ for $\left.i \neq j\right)$, $\mathbf{c}$ mixed stress induced anisotropy and inherent crossanisotropy, $\mathbf{d}$ situation with symmetry axis of inherent cross-anisotropy rotated relatively to the vertical direction

the cross-anisotropic description is horizontal excluding the case of Opalinus Clay. For details on the considered tests, the reader is referred to the original papers.

As the main calibration tool to obtain the values of model parameters, FindMinimum function was used that is available in the algebra program Mathematica [53]. The minimum of the sum of squared differences between the experimental and the model results was searched.

\subsection{Sands}

\subsubsection{Toyoura Sand}

Data on Toyoura Sand [27] involve values of $E_{v}$ and $E_{h}$. Small strain static cyclic loading in drained triaxial tests was conducted on prismatic samples. Values of the shear moduli are not provided.

Triaxial compression with $\sigma_{h}=$ const was performed on the considered reconstituted sample of Toyoura Sand, starting from the isotropic stress state at $p=100 \mathrm{kPa}$ up to the stress ratio $K=0.29$. The sample was unloaded to the initial isotropic state along the same stress path and triaxial extension with $\sigma_{v}=$ const up to $K=3.5$ followed. Cyclic loading was performed in the vertical and horizontal directions at isotropic and various anisotropic stress states during both triaxial compression and extension.

Experimental results and simulations with the calibrated model parameters are shown in Fig. 13. Values of $E_{v}$ and $E_{h}$ in the left hand side of Fig. 13 were divided by the void ratio function $f(e)=(2.17-e)^{2} /(1+e)$ [24]. In the cases of $K \leq 0.4$ and $K \geq 2.5$, a decrease in the experimental values of the Young's moduli can be seen, which corresponds to the excess of the elastic range. Hence, these data were not considered during calibration of the model parameters. Values of the model parameters are shown in the right hand side plot.

\subsubsection{Ham River Sand}

Static cyclic loading and bender element triaxial tests were conducted on reconstituted Ham River Sand samples [34]. The results provide values of $E_{v}, E_{h}, G_{v h}$ and $G_{h h}$ for various testing conditions.

Considered are the results of drained tests. Samples were normally consolidated under $K=0.45$. The experimental data obtained for different values of $p$ and the 

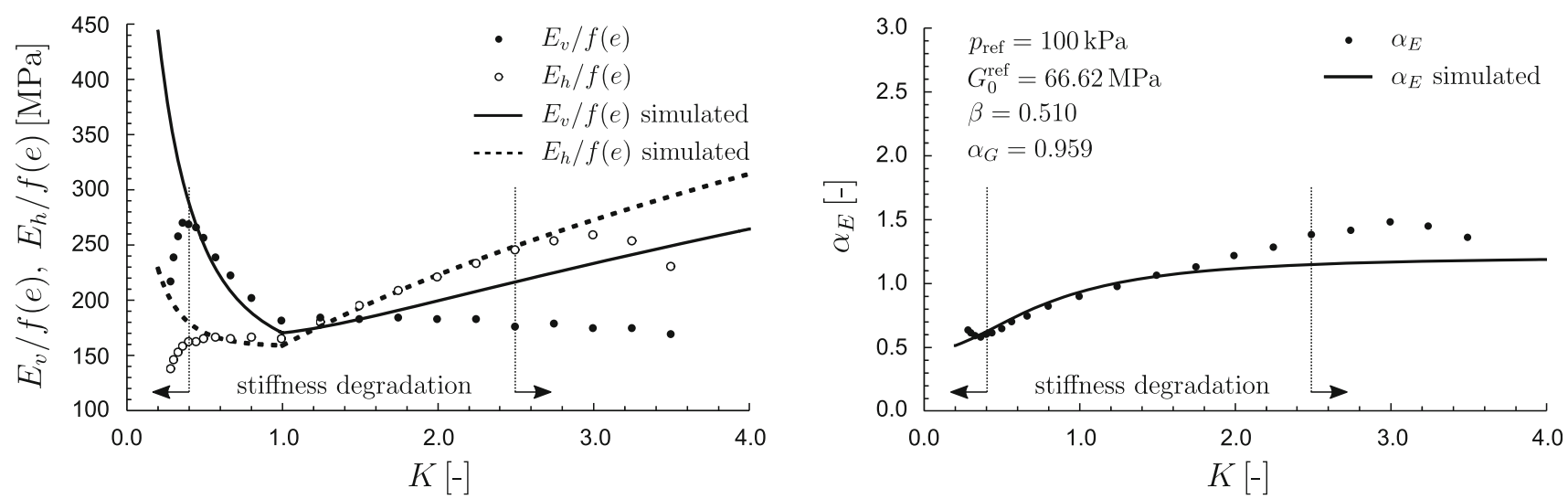

Fig. 13 Experimental [27] and simulated small strain stiffness parameters for Toyoura Sand. Left hand side: $E_{v}$ and $E_{h}$ divided by the void ratio function from [24], i.e. $E_{v} / f(e)$ and $E_{h} / f(e)$, respectively, plotted for different $K$. Right hand side: $\alpha_{E}$ plotted for different $K$. Data corresponding to $K \leq 0.4$ and $K \geq 2.5$ were not considered during calibration of parameters
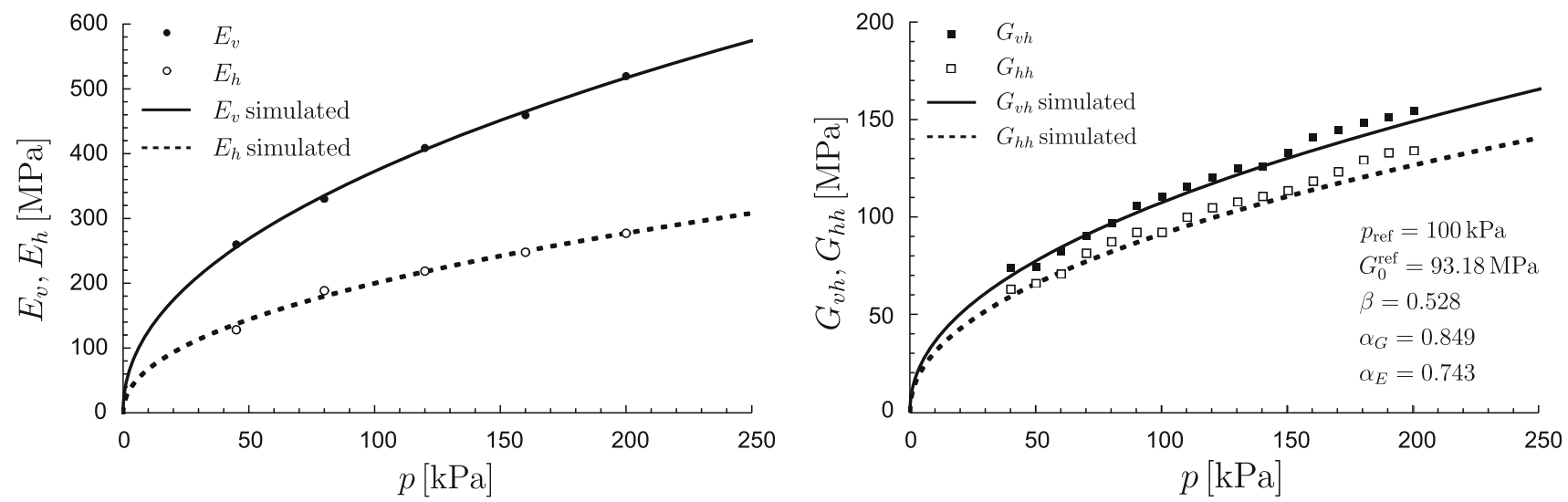

Fig. 14 Experimental [34] and simulated small strain stiffness parameters for Ham River Sand. Left hand side: $E_{v}$ and $E_{h}$ plotted for different $p$. Right hand side: $G_{v h}$ and $G_{h h}$ plotted for different $p$

corresponding simulations are presented in Fig. 14. Due to constant value of the stress ratio $K=$ const, the simulated anisotropy coefficient $\alpha_{E}=$ const . Values of the model parameters are shown in the right hand side plot.

\subsection{Clays}

\subsubsection{London Clay}

Data on the small strain stiffness parameters were obtained with the cyclic loading and bender element triaxial tests performed on the intact samples of London Clay [19].

Samples taken from different depths were consolidated to the in situ stress states. Both the drained Young's moduli and the shear moduli investigated at three different levels of $p$, representing the in situ stress, are regarded in this study. Each of the three different values of $p$ corresponds to different value of the stress ratio $K$.
The experimental values of $E_{v}, E_{h}, G_{v h}$ and $G_{h h}$ and the simulations are shown in Fig. 15. Stress ratios $K$ corresponding to the three different stress levels and values of the model parameters are all given in the right hand side plot. Points corresponding to each pair of $p$ and $K$ are denoted in the simulated plots with the bigger indicators.

\subsubsection{Gault, Kimmeridge, Oxford and London Clays}

Experimental results referred in [8] provide data on $E_{v}, E_{h}$, $G_{v h}$ and $G_{h h}$ for four different clays. Elastic stiffness parameters were investigated on both intact and reconstituted samples of Gault, Kimmeridge, Oxford and London Clays with the drained static probes and bender element triaxial tests.

All samples were tested under nearly in situ stress conditions. Values of the small strain stiffness parameters obtained for each clay and the simulated curves are plotted in Fig. 16. Stress ratios $K$ corresponding to different stress 

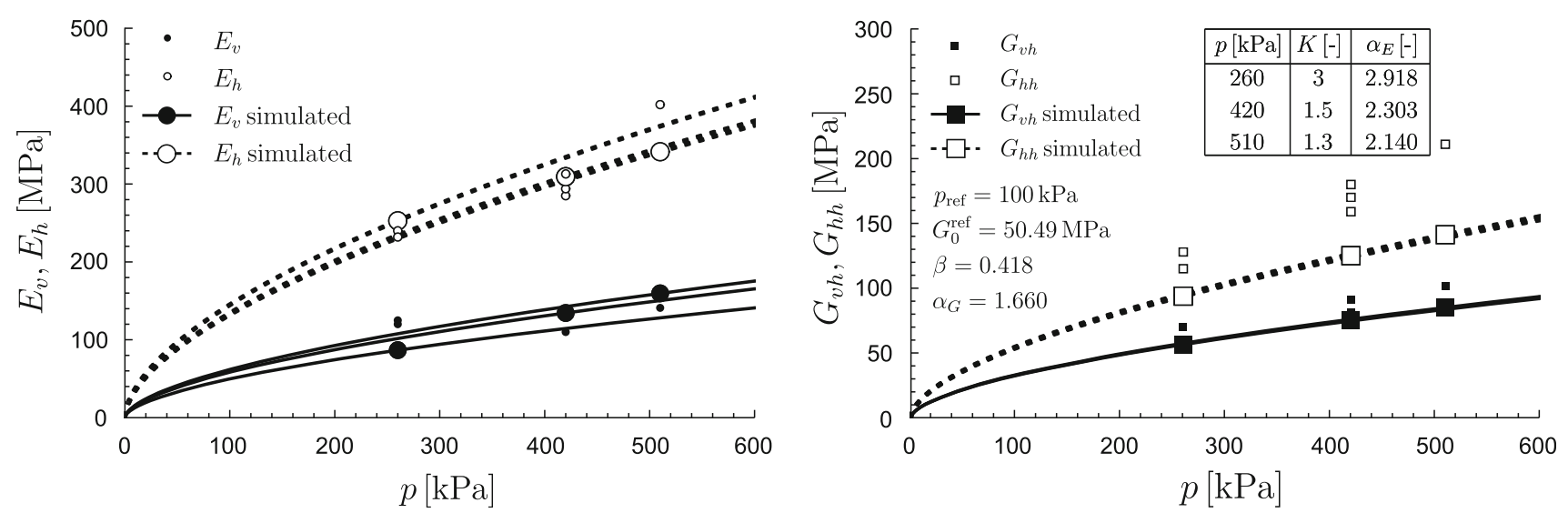

Fig. 15 Experimental [19] and simulated small strain stiffness parameters for London Clay. Left hand side: $E_{v}$ and $E_{h}$ plotted for different values of both, $p$ and $K$. Right hand side: $G_{v h}$ and $G_{h h}$ plotted for different values of both $p$ and $K$
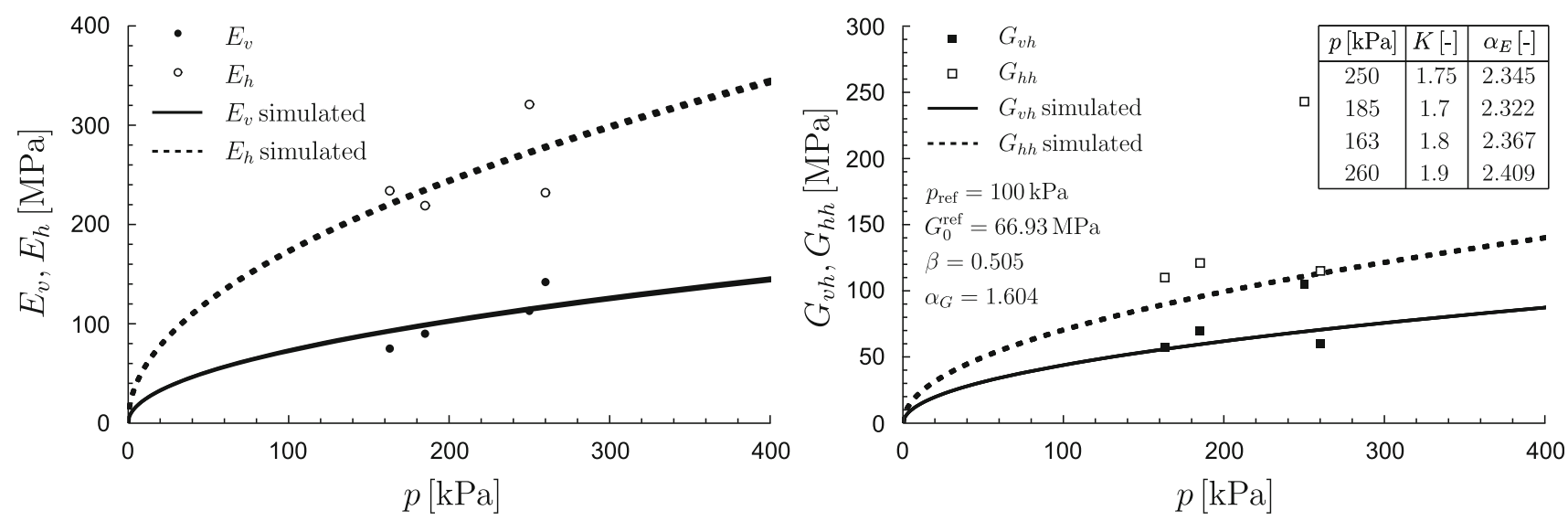

Fig. 16 Experimental [8] and simulated small strain stiffness parameters for Gault, Kimmeridge, Oxford and London Clays. Left hand side: $E_{v}$ and $E_{h}$ plotted for different values of both $p$ and $K$. Right hand side: $G_{v h}$ and $G_{h h}$ plotted for different values of both $p$ and $K$

levels and values of the model parameters are all given in the right hand side plot. Because the values of $K$ do not differ significantly, the modelled curves almost overlay each other. Hence, no indicators of different pairs of $p$ and $K$ are shown in the corresponding simulated plots.

\subsubsection{Opalinus Clay}

Anisotropic behaviour of Opalinus Clay was investigated on intact samples tested perpendicular ( $\mathrm{S}$-specimens) and parallel (P-specimens) to bedding plane in the triaxial apparatus [14, 48]. Additionally, data on samples with bedding orientation inclined at $45^{\circ}$ with respect to the loading direction (Z-specimens) are provided in [48]. The resolution of drained triaxial compression curves $\left(\varepsilon_{1}-q\right)$ presented in the referenced reports is not sufficient to focus on the details of small strain behaviour. However, the qualitative simulation of initial stiffness for $\mathrm{P}, \mathrm{Z}$ and $\mathrm{S}$ specimens could be conducted and only the initial linear segments of compression curves are considered.
In the cross-anisotropic material description of P-specimens and Z-specimens, the symmetry axis and the plane of isotropy is not collinear with vertical and horizontal directions, respectively. Hence, one needs to distinguish between the principal axes of stress and of microstructure.

Samples reported in [14] were consolidated isotropically to three different levels of $p$, i.e. 2, 5, $10 \mathrm{MPa}$ and 2, 5, 12 $\mathrm{MPa}$, in the case of $\mathrm{P}$ - and S-specimens, respectively. This was followed by the drained triaxial compression. Experimental and simulated relations between deviatoric stress $q$ and axial strain $\varepsilon_{1}$ for both $\mathrm{P}$ - and $\mathrm{S}$-specimens are shown in Fig. 17. Solely the initial small strain elastic range is considered. Values of the model parameters are shown in the upper right hand side plot. In Fig. 17, only one Pspecimen is considered, on which a multistage test was conducted. Due to lack of the experimental data for $p=10$ MPa and $p=12 \mathrm{MPa}$ in the case of S- and P-specimen, respectively, only the simulated relation is plotted.

In Fig. 18, experimental and simulated values of the corresponding Young's moduli and $\alpha_{E}$ are presented. Since 

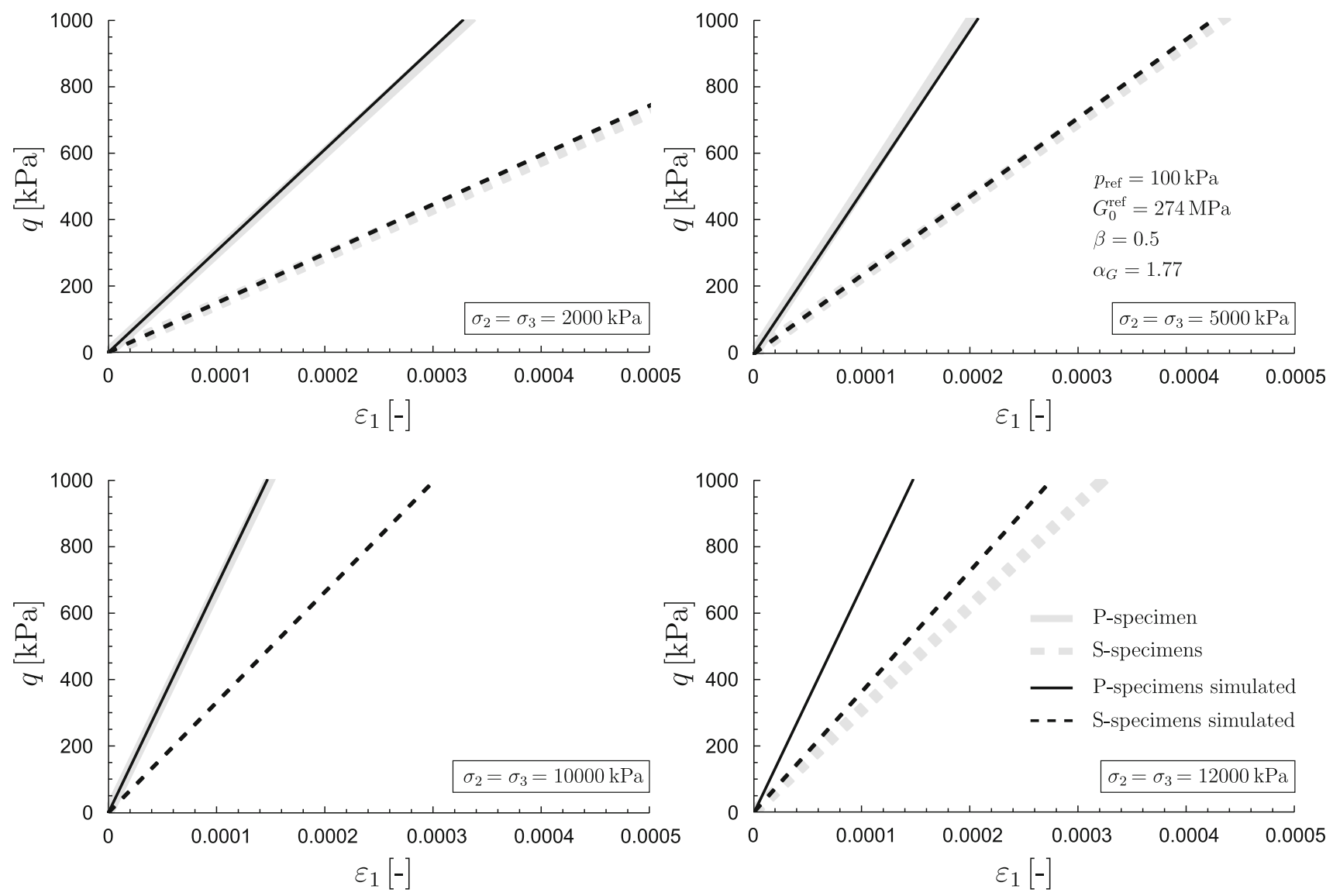

Fig. 17 Experimental [14] and simulated relation between $q$ and $\varepsilon_{1}$ for Opalinus Clay. Data are presented for P-specimen and S-specimens under different values of $\sigma_{2}=\sigma_{3}$. Only the initial linear segments of compression curves are shown due to limited accuracy of the tests within the small strain region
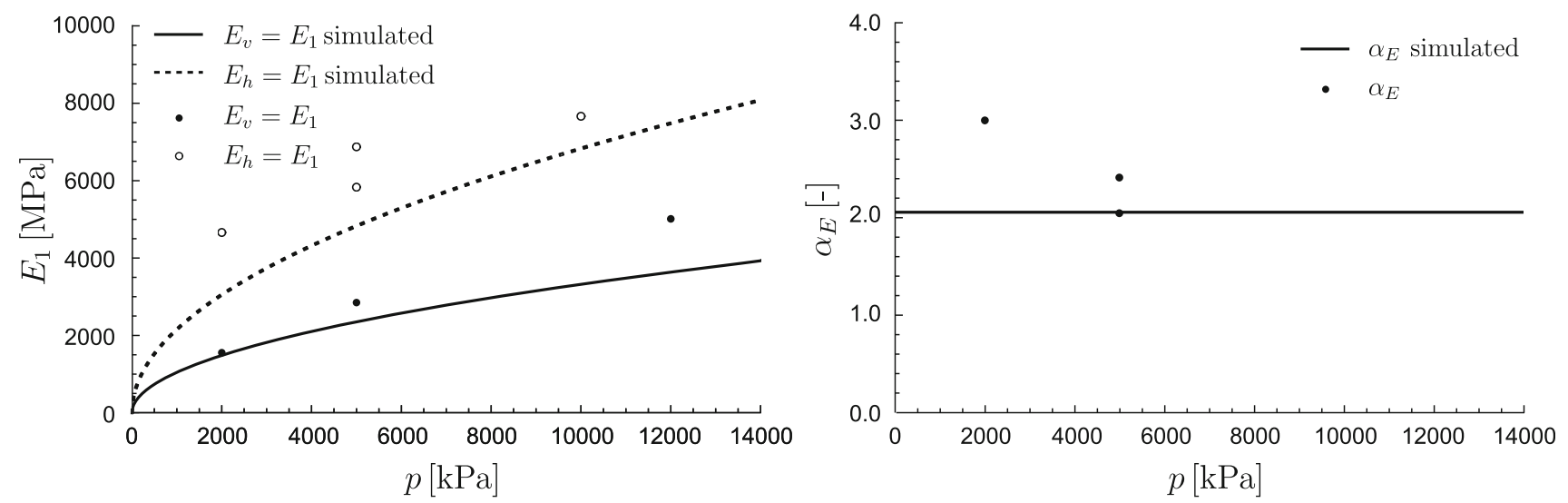

Fig. 18 Experimental [14] and simulated elastic stiffness parameters for Opalinus Clay. Left hand side: $E_{1}$ plotted for different values of $p$ equal to confining stress. $E_{1}$ obtained for P-specimens and S-specimens corresponds to $E_{h}$ and $E_{v}$, respectively. Right hand side: $\alpha_{E}$ plotted for different values of $p$

the samples were tested parallel and perpendicular to bedding, vertical Young's moduli $E_{1}$ can be interpreted as $E_{v}$ and $E_{h}$ in the case of $\mathrm{S}$-specimens and P-specimens, respectively. The modelled data were calculated with the values of parameters shown in the upper right hand side of Fig. 17. As already mentioned, the simulations were conducted only for the small strain elastic region. However, the experimental Young's moduli in Fig. 18 concern 


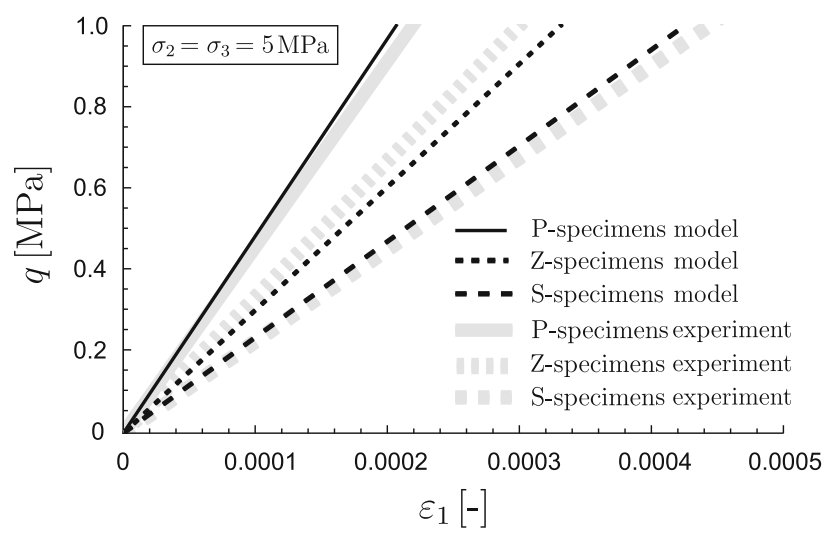

Fig. 19 Experimental [48] and simulated relation between $q$ and $\varepsilon_{1}$ for Opalinus Clay. Data are presented for P-specimens, Z-specimens and S-specimens. Only the initial linear segments of compression curves are shown due to limited accuracy of the tests within the small strain region

different ranges of strain than shown in Fig. 17. These were derived from the linear part of $q-\varepsilon_{1}$ unloading-reloading loops for S-specimens and from the initial part of $q-\varepsilon_{1}$ plot up to $25 \%$ of the peak shear strength for P-specimens.

Results analogous to these in Fig. 17 but regarding also Z-specimens are shown in Fig. 19 for selected data from [48]. Same set of model parameters as in Fig. 17 was used in all simulations considering Opalinus Clay.

\section{Conclusions}

A simple anisotropic hyperelastic model is presented in this paper. It is a refined version of the basic hyperelastic model proposed by Vermeer [51]. The refinement concerns incorporation of inherent cross-anisotropy with the mixed stress-microstructure invariant. Properties of the obtained anisotropic hyperelastic stiffness are investigated and compared with laboratory results from literature. The current experimental evidence is still limited in many aspects of small strain anisotropic behaviour of soils, and further investigations are needed. However, the proposed model is capable of a robust description of the pure inherent crossanisotropy and the mixed anisotropy observed in various tests conducted on both sands and clays. Due to the small number of parameters that can be easily related to laboratory results, the model proves to be a simple tool allowing reproduction of the small strain mixed anisotropy, being the superposition of stress-induced and inherent anisotropy as observed in natural soils.

The main application of the model can be its incorporation into some more advanced hyperelastic-plastic models to properly simulate the initial small strain stiffness and the pre-failure undrained behaviour. The further works related to the proposed model are considered, and they will concern its FE implementation within existing elastoplastic models by substitution of the popular isotropic hypoelastic formulation, e.g. [12].

Open Access This article is licensed under a Creative Commons Attribution 4.0 International License, which permits use, sharing, adaptation, distribution and reproduction in any medium or format, as long as you give appropriate credit to the original author(s) and the source, provide a link to the Creative Commons licence, and indicate if changes were made. The images or other third party material in this article are included in the article's Creative Commons licence, unless indicated otherwise in a credit line to the material. If material is not included in the article's Creative Commons licence and your intended use is not permitted by statutory regulation or exceeds the permitted use, you will need to obtain permission directly from the copyright holder. To view a copy of this licence, visit http://creativecommons. org/licenses/by/4.0/.

List of symbols $\mathrm{e}^{\mathrm{e}}, e_{i j}^{\mathrm{e}}$ : Deviatoric part of the elastic strain tensor, $e_{i j}^{\mathrm{e}}=\varepsilon_{i j}^{\mathrm{e}}-\frac{1}{3} \varepsilon_{\mathrm{V}}^{\mathrm{e}} \delta_{i j} ; \mathbf{C}, C_{i j k l}$ : Fourth-order elastic compliance tensor;

D, $D_{i j k l}$ : Fourth-order elastic stiffness tensor; $E$ : Young's modulus; $G$ : Shear modulus; $K$ : Stress ratio, $K=\sigma_{h} / \sigma_{v}$ or $K=\sigma_{3} / \sigma_{1} ; m$ : Order of the stress dependency of stiffness, in Vermeer's model $m=1-\beta$; $\mathbf{M}, M_{i j}$ : Microstructure tensor; $p$ : Mean effective stress, $p=-\frac{1}{3} \sigma_{k k}$; $q$ : Invariant of the deviatoric part of the stress tensor, $q=\sqrt{\frac{3}{2} s_{i j} s_{i j}}$; $\mathbf{s}, s_{i j}$ : Deviatoric part of the stress tensor, $s_{i j}=\sigma_{i j}+p \delta_{i j} ; \mathbf{v}, v_{i}$ : Unit vector defining the symmetry axis in a cross-anisotropic material; $W$ : Elastic potential function, $W\left(\varepsilon^{e}\right)-$ Helmholtz free energy function $W(\boldsymbol{\sigma})$-negative Gibbs free energy function; $x_{G E}, x_{G v}$ : Anisotropy exponents, $\alpha_{G}=\alpha_{E}^{x_{G E}}, \alpha_{G}=\alpha_{v}^{x_{G v}} ; \alpha$ : Anisotropy coefficient in Graham and Houlsby model, $\alpha=\sqrt{\frac{E_{h}}{E_{v}}}=\frac{G_{h h}}{G_{v h}}=\frac{v_{h h}}{v_{v h}} ; \alpha_{E}, \alpha_{G}, \alpha_{v}$ : Refined anisotropy coefficients, $\alpha_{E}=\frac{E_{h}}{E_{v}}, \alpha_{G}=\frac{G_{h h}}{G_{v h}}, \alpha_{v}=\frac{v_{h h}}{v_{v h}} ; \beta$ : Parameter coupling the Poisson's ratio and the order of elastic stiffness stress dependency in Vermeer's model; $\boldsymbol{\delta}, \delta_{i j}$ : Kronecker's symbol; $\boldsymbol{\varepsilon}^{\mathrm{e}}, \varepsilon_{i j}^{\mathrm{e}}$ : Elastic strain tensor, compression negative; $\varepsilon_{q}^{\mathrm{e}}$ : Invariant of the deviatoric part of the elastic strain tensor, $\varepsilon_{q}^{\mathrm{e}}=\sqrt{\frac{2}{3} e_{i j}^{\mathrm{e}} e_{i j}^{\mathrm{e}}} ; \varepsilon_{\mathrm{V}}^{\mathrm{e}}$ : Elastic volumetric strain, $\varepsilon_{\mathrm{V}}=\varepsilon_{i i} ; v$ : Poisson's ratio; $\sigma, \sigma_{i j}$ : Effective Cauchy stress tensor, compression negative; $\sigma_{1}, \sigma_{2}, \sigma_{3}$ : Major, intermediate and minor principal stress components,

respectively; ()$_{0},()^{0}$ : Initial values; ()$^{\mathrm{e}}$ : Elastic values; ()$_{\text {ref }},()^{\text {ref }}$ : Reference values; ()$^{\mathrm{s}},()^{\mathrm{t}}$ : Secant and tangential operators, respectively; ()$_{v},()_{h}$ : Cross-anisotropic components along the symmetry axis and in the plane of isotropy, respectively, usually collinear with vertical and horizontal directions; $(\overline{)}$ : Functions of both stress and jointed stress-microstructure invariants; $\because$ Single contraction, $\mathbf{a} \cdot \mathbf{b}=a_{i} b_{i}$ or $\mathbf{A} \cdot \mathbf{b}=A_{i j} b_{j} ;::$ : Double contraction, D : $\boldsymbol{\sigma}=D_{i j k l} \sigma_{k l}$ or $\boldsymbol{\sigma}: \boldsymbol{\sigma}=\sigma_{i j} \sigma_{i j} ; \otimes$ : Dyadic or outer product, $\mathbf{A}=\mathbf{a} \otimes \mathbf{b}=a_{i} b_{j}=A_{i j} ;\|\|$ : Euclidean norm, $\|\mathbf{x}\|=\sqrt{x_{i} x_{i}}$ or $\|\boldsymbol{\sigma}\|=\sqrt{\sigma_{i j} \sigma_{i j}} ; \operatorname{tr}()$ : Trace of a tensor, $\operatorname{tr} \boldsymbol{\sigma}=\sigma_{i i} ;()^{n}$ : Power of a tensor, $\boldsymbol{\sigma}^{n}=\overbrace{\boldsymbol{\sigma} \cdot \boldsymbol{\sigma} \cdot \ldots \cdot \boldsymbol{\sigma}}^{n}$ or $\operatorname{tr} \boldsymbol{\sigma}^{3}=\operatorname{tr}\left(\sigma_{i k} \sigma_{k l} \sigma_{l j}\right)=\sigma_{i k} \sigma_{k l} \sigma_{l i}$ 


\section{References}

1. Addenbrooke TI, Potts DM, Puzrin AM (1997) The influence of pre-failure soil stiffness on the numerical analysis of tunnel construction. Géotechnique 47(3):693-712

2. Amorosi A, Rollo F, Houlsby GT (2020) A nonlinear anisotropic hyperelastic formulation for granular materials: comparison with existing models and validation. Acta Geotech 15(1):179-196

3. Avgerinos V, Potts DM, Standing JR (2016) The use of kinematic hardening models for predicting tunnelling-induced ground movements in London Clay. Géotechnique 66(2):106-120

4. Bellotti R, Jamiolkowski M, Lo Presti DCF, O’Neill D (1996) Anisotropy of small strain stiffness in ticino sand. Géotechnique 46(1):115-131

5. Boehler JP (1979) A simple derivation of representations for nonpolynomial constitutive equations in some cases of anisotropy. Zeitschrift für Angewandte Mathematik und Mechanik, ZAMM 59(4):157-167

6. Boehler JP, Sawczuk A (1970) Equilibre limite des sols anisotropes. Journal de Mecanique 3:5-33

7. Boehler JP, Sawczuk A (1977) On yielding of oriented solids. Arch Mech 27:185-206

8. Brosse A, Hosseini Kamal R, Jardine RJ, Coop MR (2017) The shear stiffness characteristics of four Eocene-to-Jurassic UK stiff clays. Géotechnique 67(3):242-259

9. Cho W, Finno RJ (2010) Stress-strain responses of block samples of compressible Chicago glacial clays. J Geotech Geoenviron Eng 136(1):178-188

10. Cudny M (2013) Some aspects of the constitutive modelling of natural fine grained soils. Habilitation Monograph, Faculty of Civil and Environmental Engineering, Gdańsk University of Technology, Wydawnictwo IMOGEOR

11. Cudny M, Partyka E (2017) Influence of anisotropic stiffness in numerical analyses of tunneling and excavation problems in stiff soils. In: Lee W, Lee JS, Kim HK, Kim DS (eds) Proceedings of the 19th international conference on soil mechanics and geotechnical engineering, ISSMGE, Seoul, vol 2, pp 719-722

12. Cudny M, Truty A (2020) Refinement of the Hardening Soil model within the small strain range. Acta Geotech 15(8):2031-2051

13. Danne S, Hettler A (2015) Experimental and numerical strainresponse-envelopes for granular soils: performance of different constitutive models for monotonous and lo-cycle loading. In: Winter E Smith, Toll (eds) Proceedings of the XVI ECSMGE, geotechnical engineering for infrastructure and Development, ICE, Edinburgh, pp 3905-3910

14. Favero V, Ferrari A, Laloui L (2018) Anisotropic behaviour of Opalinus Clay through consolidated and drained triaxial testing in saturated conditions. Rock Mech Rock Eng 51:1305-1319

15. Fioravante V, Capoferri R (2001) On the use of multi-directional piezoelectric transducers in triaxial testing. Geotech Test $\mathrm{J}$ 24(3):243-255

16. Franzius J, Potts D, Burland JB (2005) The influence of soil anisotropy and $K_{0}$ on ground surface movements resulting from tunnel excavation. Géotechnique 55(3):189-199

17. Gajo A (2010) Hyperelastic modelling of small-strain stiffness anisotropy of cyclically loaded sand. Int J Numer Anal Meth Geomech 34:111-134

18. Gajo A, Bigoni D (2008) A model for stress and plastic strain induced nonlinear, hyperelastic anisotropy in soils. Int J Numer Anal Meth Geomech 32:833-861

19. Gasparre A (1990) Advanced laboratory characterisation of London Clay. PhD thesis, Imperial College, London

20. Gasparre A, Nishimura S, Minh NA, Coop MR, Jardine RJ (2007) The stiffness of natural London Clay. Géotechnique 57(1):33-47
21. Graham J, Houlsby GT (1983) Anisotropic elasticity of a natural clay. Géotechnique 33(2):165-180

22. Gudehus G, Mašín D (2009) Graphical representation of constitutive equations. Géotechnique 59(2):147-151

23. Hardin BO, Blandford GE (1989) Elasticity of particulate materials. J Geotech Eng Div ASCE 115(6):788-805

24. Hardin BO, Richart FEJ (1963) Elastic wave velocities in granular soils. J Soil Mech Found Div ASCE 89(SM1):33-65

25. Hashiguchi K (2018) Hypo-elastic and hyper-elastic equations of soils, short communication. Int J Numer Anal Meth Geomech 42:1554-1564

26. Hight DW, Gasparre A, Nishimura S, Minh NA, Jardine RJ, Coop MR (2007) Characteristics of the London Clay from the terminal 5 site at Heathrow Airport. Géotechnique 57(1):3-18

27. Hoque E, Tatsuoka F (2004) Effects of stress ratio on small-strain stiffness during triaxial shearing. Géotechnique 54(7):429-439

28. Houlsby GT, Amorosi A, Rojas E (2005) Elastic moduli of soils dependent on pressure: a hyperelastic formulation. Géotechnique 55(5):383-392

29. Houlsby GT, Amorosi A, Rollo F (2019) Non-linear anisotropic hyperelasticity for granular materials. Comput Geotech 115:1-11

30. Jamiolkowski M, Lancellotta R, Lo Presti DCF (1995) Remarks on the stiffness at small strains of six Italian clays. In: Shibuya M, Miura (eds) Proceedings of the first international conference on pre-failure deformation characteristics of geomaterials, Sapporo, pp 817-836

31. Janbu N (1963) Soil compressibility as determined by oedometer and triaxial test. In: Proceedings of the 3rd European conference on soil mechanics and foundation engineering, Wiesbaden, pp 19-25

32. Jovičić V, Coop MR (1998) The measurements of stiffness anisotropy in clays with bender element tests in triaxial apparatus. Geotech Test J 21(1):3-10

33. Knittel L, Wichtmann T, Niemunis A, Huber G, Espino E, Triantafyllidis T (2020) Pure elastic stiffness of sand represented by response envelopes derived from cyclic triaxial tests with local strain measurements. Acta Geotech 15(8):2075-2088

34. Kuwano R, Jardine RJ (2002) On the applicability of cross-anisotropic elasticity to granular materials at very small strains. Géotechnique 52(10):727-749

35. Lee KM, Rowe RK (1989) Deformations caused by surface loading and tunnelling: the role of elastic anisotropy. Géotechnique 39(1):125-140

36. Li Q, Ng CWW, Liu GB (2012) Determination of small-strain stiffness of Shanghai clay on prismatic soil specimen. Can Geotech J 49(8):986-993

37. Lo Presti DCF, Pallara O, Cavallaro A, Jamiolkowski M (1999) Influence of reconsolidation techniques and strain rate on the stiffness of undisturbed clays from triaxial tests. Geotech Test J 22(3):211-225

38. Mašín D, Rott J (2014) Small strain stiffness anisotropy of natural sedimentary clays: review and a model. Acta Geotech 9(2):299-312

39. McConnachie I (1974) Fabric changes in consoliated kaolin. Géotechnique 24(2):207-222

40. Mitaritonna G, Amorosi A, Cotecchia F (2014) Experimental investigation of the evolution of elastic stiffness anisotropy in a clayey soil. Géotechnique 64(6):463-475

41. Nemat-Nasser S, Hori M (1993) Overall properties of heterogeneous materials. Elsevier, Applied Mathematics and Mechanics

42. Ng C, Leung E, Lau C (2004) Inherent anisotropic stiffness of weathered geomaterial and its influence on ground deformations around deep excavations. Can Geotech J 41:12-24

43. Niemunis A, Cudny M (1998) On hyperelasticity for clays. Comput Geotech 23:221-236 
44. Niemunis A, Grandas Tavera C, Wichtmann T (2016) Peak stress obliquity in drained and undrained sands. Simulations with neohypoplasticity. In: Triantafyllidis T (ed) Holistic simulation of geotechnical installation processes, numerical and physical modelling, Springer, vol 80, pp 85-114

45. Ohde J (1936) Zur theorie der druckverteilung im baugrund. Der Bauingenieur 20:451-459

46. Pickering DJ (1970) Anisotropic elastic parameters for soil. Géotechnique 20(2):217-233

47. Puzrin AM, Burland JB, Strandind JR (2012) Simple approach to predicting ground displacements caused by tunnelling in undrained anisotropic elastic soil. Géotechnique 62(4):341-352

48. Salager S, Francois B, Nuth M, Laloui L (2012) Constitutive analysis of the mechanical anisotropy of Opalinus Clay. Acta Geotech 8(1):137-154

49. Schanz T, Vermeer PA, Bonnier PG (1999) The hardening soil model: formulation and verification. Beyond 2000 in Computational Geotechnics-10 Years of Plaxis. Balkema, Rotterdam, pp $1-16$

50. Sivakumar V, Doran IG, Graham J, Johnson A (2001) The effect of anisotropic elasticity on the yielding characteristics of overconsolidated natural clay. Can Geotech J 38:125-137
51. Vermeer PA (1985) A five constant model unifying well established concepts. In: Gudehus D, Vardoulakis (eds) Constitutive relations of soils, Balkema, Rotterdam, pp 175-197

52. Waffenschmidt T, Menzel A, Kuhl E (2012) Anisotropic density growth of bone: a computational micro-sphere approach. Int $\mathbf{J}$ Solids Struct 49:1928-1946

53. Wolfram Research Inc (2017) Mathematica 11

54. Xiao Y, Zhang Z, Wang J (2020) Granular hyperelasticity with inherent and stress-induced anisotropy. Acta Geotech 15(3):671-680

55. Yong RN, Silvestri V (1979) Anisotropic behaviour of a sensitive clay. Can Geotech J 16(2):335-350

56. Zwanenburg C (2005) The influence of anisotropy on the consolidation behaviour of peat. PhD thesis, Delft University of Technology

Publisher's Note Springer Nature remains neutral with regard to jurisdictional claims in published maps and institutional affiliations. 